\title{
Forward dihadron correlations in deuteron-gold collisions with a Gaussian approximation of JIMWLK
}

\author{
T. Lappi ${ }^{1,2}$ and H. Mäntysaari ${ }^{1}$ \\ 1 Department of Physics, P.O. Box 35, 40014 University of Jyväskylä, Finland \\ ${ }^{2}$ Helsinki Institute of Physics, P.O. Box 64, 00014 University of Helsinki, Finland
}

\begin{abstract}
We compute dihadron correlations in forward deuteron-gold or proton-gold collisions. The running coupling BK equation is used to calculate the energy dependence of the dipole cross sections and extended to higher-point Wilson line correlators using a factorized Gaussian approximation. Unlike some earlier works we include both the "inelastic" and "elastic" contributions to the dihadron cross section. We show that the double parton scattering contribution is included in our calculation and obtain both an away side peak that roughly agrees with experimental observations and an estimate for the azimuthal angle-independent pedestal. We find that nonlinear effects for momenta close to the saturation scale are clearly visible in the away side peak structure

PACS numbers: $13.85 . \mathrm{Hd}, 25.75 . \mathrm{Gz}, 24.85 .+\mathrm{p}$
\end{abstract}

\section{INTRODUCTION}

At high energy or, equivalently small $x$, the interactions of hadrons are expected to be dominated by nonlinear strong color fields. A convenient effective theory approach to studying these color fields is provided by the Color Glass Condensate (for reviews see e.g. 1, 2]). This effective theory is based on the division of the QCD degrees of freedom into a static (in light cone time) large $x$ part, which is described as a color source, and the small $x$ gluons as a color field radiated by these sources. The rapidity scale separating the two is, of course, arbitrary, and should be chosen to correspond to the rapidity scale probed in the particular physical process being looked at. From the requirement that physical cross sections must not depend on this arbitrary scale one can derive renormalization group equations describing the rapidity dependence of the probability distribution of the color sources. The general, leading $\ln x$, equation for the probability distribution of different color charge configurations is known by the acronym JIMWLK (see Ref. 3] and references therein). The mean field approximation of JIMWLK, known as the Balitsky-Kovchegov [4-6] equation, is widely used in phenomenological applications. The BK equation describes the rapidity dependence of the unintegrated gluon distribution and can loosely be thought of as describing the rapidity dependence of the mean number of gluons instead of the whole probability distribution encoded in the JIMWLK equation.

The BK equation is usually enough when studying single inclusive cross sections in a dilute-dense collision such as DIS or proton-nucleus collisions at forward rapidities. Understanding the initial state of a heavy-ion collision, on the other hand, necessitates the, eventually numerical, solution of a much more complicated interacting system [7, 8. Therefore these very inclusive quantities do not provide direct experimental access to all of the physics described by the JIMWLK equation (for a discussion of potential observables in diffractive DIS that go beyond the mean-field BK approximation see e.g. Ref. [9]).
It has recently become more evident that multiparticle correlations could provide precisely such an observable.

Quite generically multiparton correlations are more sensitive to the detailed dynamics of the colliding objects than single particle distributions. An attractive observable, measurable at both RHIC and the LHC, are dihadron correlations in the forward rapidity region in proton-nucleus or deuteron-nucleus collisions. In this kinematical regime the dilute and relatively well understood large $x$ part of the proton provides with a calibrated probe of the nonlinear small $x$ gluon fields in the target nucleus. By varying the rapidity one can study different values of $x$ and the transverse momentum dependence of the correlation should be directly sensitive to the dominant intrinsic transverse momentum scale in the target, the saturation scale $Q_{\mathrm{s}}$.

Indeed measurements of correlations between two forward dihadrons measured in dAu collisions at RHIC [10, 11 seem to show indications of "initial state" or "cold nuclear matter" effects that are significantly stronger than in pp collisions or at central rapidities 12 14. The upcoming LHC proton-lead collisions will provide more opportunities to study these phenomena in a wider kinematical range. These observations have provided an impetus for renewed interest in the gluonic correlations included in the JIMWLK evolution [15 21. In particular it was argued [17-19] that the result of a full JIMWLK evolution, also at finite $N_{\mathrm{c}}$, can quite accurately be captured by the so called Gaussian approximation, relating higher-point Wilson line correlators to the two-point function. Thus in the Gaussian approximation one can construct observable cross sections using the solution of the BK equation alone, which is much more convenient in practice than solving the full JIMWLK equation. These recent theoretical developments were not fully reflected in the pioneering calculations of dihadron correlations in [22, 23] (see also the more recent work [24, 25] where a $k_{T}$-factorized approximation is derived in a certain kinematical limit). The main purpose of this paper is to implement the Gaussian approximation, which so far has 
only been tested for particular coordinate space configurations, in a full calculation of the dihadron correlation

We shall first, in Sec. II. discuss the description of the single inclusive hadron spectrum as a baseline that should be consistently described by the same calculation and used to constrain the initial conditions of evolution in rapidity. We then, in Sec. III, recall the expression of the dihadron cross section derived in Ref. 22 using the light cone perturbation theory formalism. In Sec. IV we show that the dihadron cross section as derived in Ref. 22 contains a logarithmically infrared divergent part, which has been overlooked in the previous literature. We identify this as a double parton scattering contribution describing the independent scattering of two partons already present in the dilute projectile. The double parton scattering contribution must be consistently subtracted from the correlated cross section and absorbed into a separate contribution that depends on additional nonperturbative information about the projectile in the form of a double parton distribution. We then discuss in Sec. V the expressions for the Wilson line correlators used in this work. In Sec. VI we present our results for the dihadron cross section. We show that using the full expression for the dihadron cross section enhances the away-side peak by a large factor compared to approximations used in the previous literature. The qualitative features of the results in the previous literature remain, most prominently a strong dependence of the correlation on $p_{T} / Q_{\mathrm{s}}$, which leads to a large difference between deuteron-gold and proton-proton collisions. Technical details on the DPS limit are relegated to Appendix $\mathrm{A}$ and on the impact parameter dependence to Appendix B.

\section{BASELINE DESCRIPTION OF THE TARGET: BK EVOLUTION AND SINGLE INCLUSIVE SPECTRA}

We shall here use the dipole cross sections obtained from solving numerically the BK evolution equation using the Balitsky running coupling prescription 28] (see the comparison of different running coupling schemes in Ref. [29]). The evolution equations must be supplemented with an initial condition at a starting rapidity (or $x$ ). Ideally one would like to fully constrain the initial conditions of BK/JIMWLK evolution by a comparison to small $x$ DIS data. Due to the large amount of precise data available from HERA, the expectation value of the dipole cross section in a proton is quite well constrained. Several ways to extend these parametrization to nuclei exist in the literature, and in practice it is useful to also compare with single inclusive hadron production data in $\mathrm{pA}$ and $\mathrm{dAu}$ to further constrain the nuclear dipole cross sections. We shall here first recall the ("hybrid formalism") equations used to calculate the single inclusive hadron yields at forward rapidity. We then discuss the MV model parametrization of the initial condition used in this paper and compare it to the experimental forward

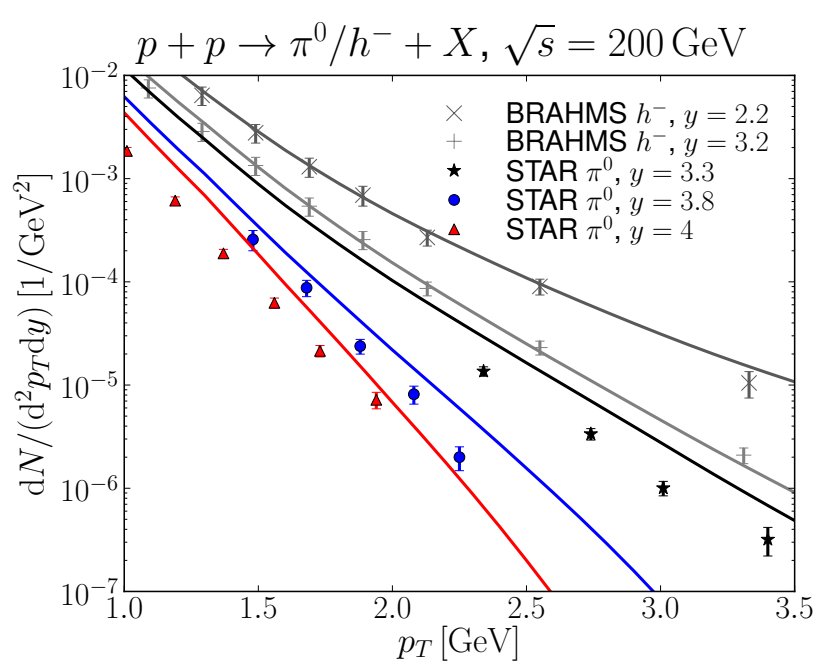

FIG. 1: The identified hadron spectra at forward rapidity compared to the experimental data from proton-proton collisions from the STAR [26] and BRAHMS 27] collaborations.

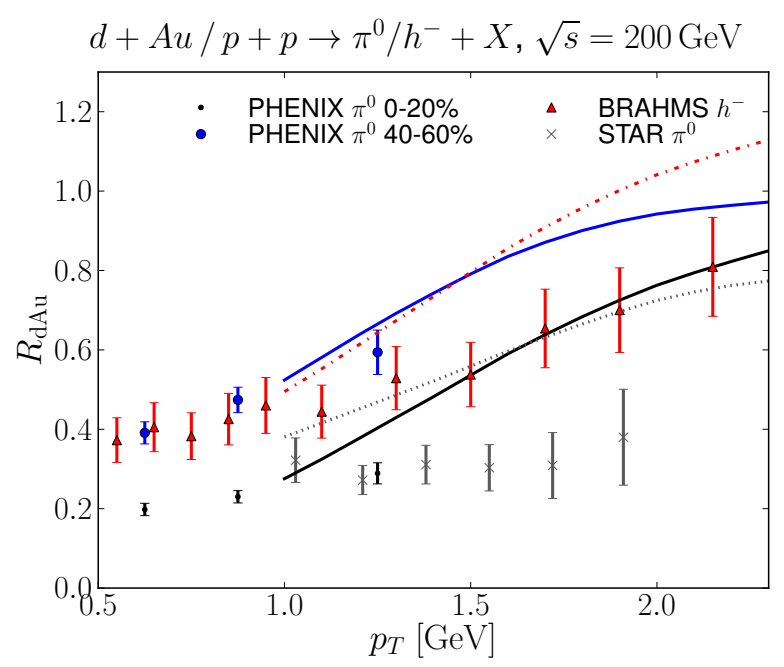

FIG. 2: The nuclear modification factor $R_{\mathrm{dAu}}$ using our MV dipole cross section parametrization. The experimental results shown are PHENIX 11] centrality dependent (solid curves, $3<y<3.8$ ) and BRAHMS [27] ( $y=3.2$, upper dashed line) and STAR [26] $(y=4$, lower dashed line) minimum bias results. The calculations are the spectra obtained using Eq. 3.

single inclusive spectra.

The single inclusive yields for quark and gluon production are given by 30 32.

$$
\begin{aligned}
\frac{\mathrm{d} N^{h A \rightarrow q X}}{\mathrm{~d} y \mathrm{~d}^{2} \mathbf{q}_{T}} & =\frac{1}{(2 \pi)^{2}} x q(x) S\left(\mathbf{q}_{T}\right), \\
\frac{\mathrm{d} N^{h A \rightarrow g X}}{\mathrm{~d} y \mathrm{~d}^{2} \mathbf{q}_{T}} & =\frac{1}{(2 \pi)^{2}} x g(x) \widetilde{S}\left(\mathbf{q}_{T}\right),
\end{aligned}
$$


where $S\left(\mathbf{q}_{T}\right)$ is the Fourier-transform of the fundamental representation dipole operator $\langle\hat{D}\rangle$ and $\widetilde{S}\left(\mathbf{q}_{T}\right)$ the corresponding quantity in the adjoint representation. Here $x q(x)$ and $x g(x)$ are standard collinear parton distribution functions describing the large $x$ partons from the dilute projectile, for which we use the CTEQ NLO 33. parametrization. To get the single hadron spectrum we convolute the parton-level cross section with the DSS [34] fragmentation function, resulting in

$$
\frac{\mathrm{d} N^{h A \rightarrow h^{\prime} X}}{\mathrm{~d} y \mathrm{~d}^{2} \mathbf{q}_{T}}=\sum_{i} \int \frac{\mathrm{d} z}{z^{2}} \frac{\mathrm{d} N^{h A \rightarrow i X}}{\mathrm{~d} y \mathrm{~d}^{2}\left(\mathbf{q}_{T} / z\right)} D_{i \rightarrow h^{\prime}}\left(z, \mu^{2}\right) .
$$

Throughout this work the scale $\mu$ at which the parton distribution function, fragmentation function and strong coupling constant $\alpha_{\mathrm{s}}$ are evaluated is chosen to be the transverse momentum of the produced hadron in single inclusive production and transverse momentum of the leading hadron in dihadron production.

As an initial condition we have used the MV model parameters used in Ref. 35] to fit single inclusive hadron production data at RHIC. The initial dipole cross section is

$$
S(r)_{x=x_{0}}=\exp \left\{-\frac{r^{2} Q_{\mathrm{s} 0}^{2}}{4} \ln \left(e+\frac{1}{r^{2} \Lambda_{\mathrm{QCD}}^{2}}\right)\right\},
$$

with an initial saturation scale $Q_{\mathrm{s} 0}^{2}=0.2 \mathrm{GeV}^{2}$ at $x_{0}=$ 0.007 for the proton, as in Ref. 35]. A comparison of the resulting yields in proton-proton collisions to the forward results of the STAR [26] and BRAHMS 27] experiments is shown in Fig. 1. While the description of the data is not perfect, we are confident that this is a realistic enough baseline for understanding nuclear modifications to the dihadron yield. Note that there are no arbitrary normalization $K$-factors used in this work.

Estimates based on nuclear geometry would suggest [36, 37] that the initial saturation scale $Q_{\mathrm{s} 0}^{2}$ should be even as large as $A^{1 / 3} \sim 6$ times that of the proton (for minimum bias collisions). Based on both experimental data and the general expectation that saturation effects should vanish for $p_{T} \gg Q_{\mathrm{s}}$, it would also seem natural for the nuclear modification factor $R_{\mathrm{dAu}}$ to approach 1 for high $p_{T}$ at least at midrapidity. Assuming that the transverse area of a proton also at small $x$ is identified with the total inelastic nucleon-nucleon cross section, this would happen with $Q_{\mathrm{sA}}^{2} \sim N_{\mathrm{bin}}^{\mathrm{pA}} Q_{\mathrm{s} p}^{2} \approx 3.6 Q_{\mathrm{s} p}^{2}$ for a minumum bias dAu collision ${ }^{1}$.

1 One would generically expect $Q_{\mathrm{s}}^{2}$ to be proportional to the number of overlapping nucleons, a quantity which is in practice encoded as the number of binary collisions in a Glauber model for a proton-nucleus collision. In this paper we will assume $N_{\text {bin }}^{\mathrm{pA}}=N_{\text {bin }}^{\mathrm{dA}} / 2$ for simplicity. Note that $N_{\text {bin }}^{\mathrm{pA}}$ is also the number of participant nucleons in the target nucleus in a $\mathrm{pA}$ collision.
However, fits to identified hadron spectra, especially at forward rapidities tend to favor a smaller nuclear saturation scale. In the calculation of Ref. 35, for example, a calculation of hadron spectra in deuteron-gold collisions is made with an initial nuclear saturation scale $Q_{\mathrm{sA}}^{2} \approx 2.3 Q_{\mathrm{s} p}^{2}$ (comparing $Q_{\mathrm{sA}}$ and $Q_{\mathrm{s} p}$ at $x=0.007$, taking into account the different $x_{0}$ used for protons and for nuclei). These saturation scales give a good description of the BRAHMS charged hadron spectra at $\eta=2.2$ and $\eta=3.2$.

For the most forward $\pi^{0}$ data the nuclear modification ratio $R_{\mathrm{dAu}}$ seems to decrease faster with $y$ in the data than predicted by running coupling BK evolution. For example, in the calculation of Ref. [35] the STAR very forward $\eta=4$ data requires an additional suppression by $K$-factors 0.4 for pp and 0.3 for $\mathrm{dAu}$ and a similar effect is seen in 37, 38. This is problematic for the dihadron correlation, for which the STAR forward data comes precisely at this same kinematical region.

In view of these uncertainties, we shall not attempt to provide the best possible fit to all the existing single inclusive particle production data in this work. Instead we use as an initial condition for nuclei the same parametrization (4) as for protons, with a nuclear saturation scale obtained by straightforward $N_{\text {bin }}$ scaling. This is essentially equivalent to the Monte Carlo rcBK model of Ref. 39, where the saturation scale $Q_{\mathrm{s}}^{2}$ is taken to be proportional to the number of overlapping nucleons in a position in the transverse plane. In particular it leads, by construction, to a nuclear modification factor $R_{\mathrm{dAu}}$ approaching unity at high transverse momenta, when the unintegrated parton distribution is probed at the scale of the initial condition at $x_{0}$. For minimum bias collisions this leads to an initial nuclear saturation scale $Q_{\mathrm{s} 0}^{2}=0.72 \mathrm{GeV}^{2}$, and for a $0-20 \%$ centrality class $Q_{\mathrm{s} 0}^{2}=1.51 \mathrm{GeV}^{2}$, using the $N_{\text {bin }}$ values from Ref. [11.

We show in Fig. 2 the resulting forward $\pi^{0}$ nuclear modification ratio $R_{\mathrm{dAu}}$ compared to the PHENIX 11 . centrality dependent and BRAHMS 27] and STAR 26] minimum bias data. Let us now point the reader to some features in this plot. Firstly, none of the nuclear data compared to here has been used as an input in the calculation; instead the initial condition for BK evolution has been set from a simple and straightforward $N_{\text {bin }}$ scaling of the saturation scale. Secondly, the STAR minimum bias data exhibits approximately as much suppression as the most central PHENIX data. Although the two are at slightly different rapidities, it seems quite generically impossible to simultaneously describe both data sets in a unique parametrization as simple as ours. A more detailed treatment of minimum bias collisions by averaging over different centralities in the calculation (instead of a single $Q_{\mathrm{s}}$ value characterising minimum bias collisions) could also be expected to improve this description. Note that the negative hadron nuclear modification ratio becomes $>1$ at high $p_{T}$ due to the difference between the deuteron and proton probes (a relative excess of $d$ quarks in the deuteron over the proton leads to 
an enhancement in negative particle production independently of the target). All in all, given that this is a pure prediction for the nuclear modifiction of single particle production, without arbitrary $K$ factors or adjusting the parameters to the nuclear data, we consider this as an adequate parametrization for the purposes of understanding the basic features of dihadron correlations, which is the main purpose of this paper.

\section{DIHADRON CORRELATIONS}

We shall consider the scattering process for forward dihadron production as a large $x$ quark with momen- tum $p^{+}$from the probe deuteron or proton, propagating eikonally through the target nucleus or proton. It can radiate a gluon with momentum $k^{+}=z p^{+}$and is left with a longitudinal momentum $q^{+}=(1-z) p^{+}$. In the high energy limit the scattering of both the quark and the gluon can be described by an eikonal approximation, where they pick up a phase given by a Wilson line in the color field of the target. The detailed derivation of the double inclusive cross section is performed in Ref. 22 and results in the following expression for the $q A \rightarrow q g X$ cross section:

$$
\begin{aligned}
\frac{\mathrm{d} \sigma^{q A \rightarrow q g X}}{\mathrm{~d} k^{+} \mathrm{d}^{2} \mathbf{k}_{T} \mathrm{~d} q^{+} \mathrm{d}^{2} \mathbf{q}_{T}}= & \alpha_{\mathrm{s}} C_{\mathrm{F}} \delta\left(p^{+}-k^{+}-q^{+}\right) \int \frac{\mathrm{d}^{2} \mathbf{x}_{T}}{(2 \pi)^{2}} \frac{\mathrm{d}^{2} \mathbf{x}_{T}^{\prime}}{(2 \pi)^{2}} \frac{\mathrm{d}^{2} \mathbf{b}_{T}}{(2 \pi)^{2}} \frac{\mathrm{d}^{2} \mathbf{b}_{T}^{\prime}}{(2 \pi)^{2}} e^{i \mathbf{k}_{T} \cdot\left(\mathbf{x}_{T}^{\prime}-\mathbf{x}_{T}\right)} e^{i \mathbf{q}_{T} \cdot\left(\mathbf{b}_{T}^{\prime}-\mathbf{b}_{T}\right)} \\
& \times \sum_{\alpha \beta \lambda} \phi_{\alpha \beta}^{\lambda *}\left(\mathbf{x}_{T}^{\prime}-\mathbf{b}_{T}^{\prime}\right) \phi_{\alpha \beta}^{\lambda}\left(\mathbf{x}_{T}-\mathbf{b}_{T}\right)\left\{S^{(4)}\left(\mathbf{b}_{T}, \mathbf{x}_{T}, \mathbf{b}_{T}^{\prime}, \mathbf{x}_{T}^{\prime}\right)-S^{(3)}\left(\mathbf{b}_{T}, \mathbf{x}_{T}, \mathbf{z}_{T}^{\prime}\right)\right. \\
& \left.-S^{(3)}\left(\mathbf{z}_{T}, \mathbf{x}_{T}^{\prime}, \mathbf{b}_{T}^{\prime}\right)+S^{(2)}\left(\mathbf{z}_{T}, \mathbf{z}_{T}^{\prime}\right)\right\}
\end{aligned}
$$

with $\mathbf{z}_{T}=z \mathbf{x}_{T}+(1-z) \mathbf{b}_{T}$ and likewise, $\mathbf{z}_{T}^{\prime}=z \mathbf{x}_{T}^{\prime}+(1-z) \mathbf{b}_{T}^{\prime}$. Here the target is described by expectation values of the Wilson line operators

$$
\begin{aligned}
S^{(4)}\left(\mathbf{b}_{T}, \mathbf{b}_{T}^{\prime}, \mathbf{x}_{T}, \mathbf{x}_{T}^{\prime}\right) & =\frac{2}{N_{\mathrm{c}}{ }^{2}-1}\left\langle\operatorname{Tr}\left(V\left(\mathbf{b}_{T}\right) V^{\dagger}\left(\mathbf{b}_{T}^{\prime}\right) t^{d} t^{c}\right)\left[U\left(\mathbf{x}_{T}\right) U^{\dagger}\left(\mathbf{x}_{T}^{\prime}\right)\right]^{c d}\right\rangle \\
S^{(3)}\left(\mathbf{b}_{T}, \mathbf{x}_{T}, \mathbf{z}_{T}^{\prime}\right) & =\frac{2}{N_{\mathrm{c}}{ }^{2}-1}\left\langle\operatorname{Tr}\left(V^{\dagger}\left(\mathbf{z}_{T}^{\prime}\right) t^{c} V\left(\mathbf{b}_{T}\right) t^{d}\right) U^{c d}\left(\mathbf{x}_{T}\right)\right\rangle \\
S^{(2)}\left(\mathbf{z}_{T}, \mathbf{z}_{T}^{\prime}\right) & =\frac{1}{N_{\mathrm{c}}}\left\langle\operatorname{Tr}\left(V\left(\mathbf{z}_{T}\right) V^{\dagger}\left(\mathbf{z}_{T}^{\prime}\right)\right)\right\rangle .
\end{aligned}
$$

The momenta of the produced gluon and quark are $k$ and $q$ respectively. Likewise, $\mathbf{x}_{T}, \mathbf{x}_{T}^{\prime}$ should be interpreted as the transverse position of the gluon, $\mathbf{b}_{T}, \mathbf{b}_{T}^{\prime}$ of the quark after the scattering and $\mathbf{z}_{T}, \mathbf{z}_{T}^{\prime}$ of the quark before the scattering; in the amplitude and the complex conjugate respectively. The wave function $\phi$ describes $q \rightarrow q g$ splitting in coordinate space, and its expression in the massless limit is given in Eq. (13). In our numerical calculations we set the quark mass $m_{q}=0.14 \mathrm{GeV}$, but the finite quark mass has little effect on the final results.

The scattering amplitude is a sum of two terms corresponding to the radiation of the gluon happening before and after the interaction with the target. Out of the three operators $(6), \sqrt{7}),(8)$ in $S^{(4)}$ the gluon is radiated before the interaction and in $S^{(2)}$ after, with $S^{(3)}$ corresponding to the interference between gluon radiation before and after the target.

The operators (6), (7), (8) are expressed in terms of fundamental and adjoint representation Wilson lines, denoted here by $V$ and $U$, respectively. In the following we denote the dipole and quadrupole operators by

$$
\hat{D}\left(\mathbf{x}_{T}-\mathbf{y}_{T}\right) \equiv \frac{1}{N_{\mathrm{c}}} \operatorname{Tr}\left(V\left(\mathbf{x}_{T}\right) V^{\dagger}\left(\mathbf{y}_{T}\right)\right)
$$

$$
\hat{Q}\left(\mathbf{x}_{T}, \mathbf{y}_{T}, \mathbf{u}_{T}, \mathbf{v}_{T}\right)=\frac{1}{N_{\mathrm{c}}} \operatorname{Tr}\left(V\left(\mathbf{x}_{T}\right) V^{\dagger}\left(\mathbf{y}_{T}\right) V\left(\mathbf{u}_{T}\right) V^{\dagger}\left(\mathbf{v}_{T}\right)\right)
$$

and their expectation values as $S \equiv S^{(2)} \equiv\langle\hat{D}\rangle$ and $Q \equiv\langle\hat{Q}\rangle$. In terms of these the higher-point correlators in Eq. (5) can be written in the form quoted in Refs. [15, 17]

$$
\begin{array}{r}
S^{(4)}\left(\mathbf{b}_{T}, \mathbf{b}_{T}^{\prime}, \mathbf{x}_{T}, \mathbf{x}_{T}^{\prime}\right)=\left\langle-\frac{1}{N_{\mathrm{c}}^{2}-1} \hat{D}\left(\mathbf{b}_{T}, \mathbf{b}_{T}^{\prime}\right)\right. \\
\left.+\frac{N_{\mathrm{c}}^{2}}{N_{\mathrm{c}}^{2}-1} \hat{D}\left(\mathbf{x}_{T}, \mathbf{x}_{T}^{\prime}\right) \hat{Q}\left(\mathbf{b}_{T}, \mathbf{b}_{T}^{\prime}, \mathbf{x}_{T}^{\prime}, \mathbf{x}_{T}\right)\right\rangle
\end{array}
$$




$$
\begin{aligned}
& S^{(3)}\left(\mathbf{b}_{T}, \mathbf{x}_{T}, \mathbf{z}_{T}^{\prime}\right)= \\
& \left\langle\frac{N_{\mathrm{c}}{ }^{2}}{N_{\mathrm{c}}{ }^{2}-1} \hat{D}\left(\mathbf{b}_{T}, \mathbf{x}_{T}\right) \hat{D}\left(\mathbf{x}_{T}, \mathbf{z}_{T}^{\prime}\right)-\frac{1}{N_{\mathrm{c}}{ }^{2}-1} \hat{D}\left(\mathbf{b}_{T}, \mathbf{z}_{T}^{\prime}\right)\right\rangle .
\end{aligned}
$$

We will discuss explicitly in Sec. $\mathrm{V}$ the Gaussian approximation used in this paper to obtain the Wilson line correlators in Eqs. (11) and (12). Computing the dihadron correlation in LHC kinematics will require taking into account also the gluon-initiated channel, because the probe $x$ will not be as large. This would require calculating an eight-point function of Wilson lines, which we leave for future work.

\section{DOUBLE PARTON SCATTERING CONTRIBUTION}

In the massless limit the wave function product appearing in Eq. (5) is given by

$$
\sum_{\alpha \beta \lambda} \phi_{\alpha \beta}^{\lambda *}\left(\mathbf{u}_{T}^{\prime}\right) \phi_{\alpha \beta}^{\lambda}\left(\mathbf{u}_{T}\right)=\frac{8 \pi^{2}}{k^{+}} \frac{\mathbf{u}_{T} \cdot \mathbf{u}_{T}^{\prime}}{\left|\mathbf{u}_{T}\right|^{2}\left|\mathbf{u}_{T}^{\prime}\right|^{2}}\left(1+(1-z)^{2}\right),
$$

with $\mathbf{u}_{T}=\mathbf{x}_{T}-\mathbf{b}_{T}$ and $\mathbf{u}_{T}^{\prime}=\mathbf{x}_{T}^{\prime}-\mathbf{b}_{T}^{\prime}$.

In the full dihadron cross section one must also include a "double parton scattering" (DPS) contribution that corresponds to the independent scattering of two partons from the probe. This contribution, whose importance for understanding the $\Delta \varphi$-independent pedestal of the double inclusive cross section, was emphasized in [40. We shall show that Eq. (5) contains a part of this (DPS) contribution, which must be subtracted from it to avoid double counting. This is in contrast with Ref. 24, 25 where, instead of the full cross section Eq. (5), one considers only the "correlation limit" in which the DPS contribution vanishes. As discussed in Ref. 24] the full cross section can differ significantly from the correlation limit when the transverse momenta of the produced particles are comparable to the saturation scale, which is precisely the regime that is interesting for observing the effects of large color fields in the target.

In the limit where the gluon is far away from the quark: $\left|\mathbf{b}_{T}-\mathbf{b}_{T}^{\prime}\right| \sim\left|\mathbf{x}_{T}-\mathbf{x}_{T}^{\prime}\right| \sim 1 / Q_{\mathrm{s}}, u_{T} \equiv\left|\mathbf{b}_{T}-\mathbf{x}_{T}\right| \gg 1 / Q_{\mathrm{s}}$ the Wilson lines of the quark and the gluon are uncorrelated. Therefore the expectation values in $S^{(4)}$ factorize into a product of an adjoint representation dipole at the location of the gluon and a fundamental representation one at the location of the quark. We call this kinematical regime the "DPS" limit since, as we will argue in the following, it corresponds to a quark and a gluon, present already in the wavefunction of the incoming dilute projectile, scattering independently off the target. Factorizing the expectation values and using the fact that the expectation values must be color singlets we can write $S^{(4)}$ in the DPS limit as

$$
\begin{aligned}
& S^{(4)}\left(\mathbf{b}_{T}, \mathbf{x}_{T}, \mathbf{b}_{T}^{\prime}, \mathbf{x}_{T}^{\prime}\right) \underset{\mathrm{DPS}}{\approx} S_{\mathrm{DPS}}^{(4)}\left(\mathbf{b}_{T}, \mathbf{x}_{T}, \mathbf{b}_{T}^{\prime}, \mathbf{x}_{T}^{\prime}\right) \\
\equiv & \frac{2}{N_{\mathrm{c}}^{2}-1}\left\langle\operatorname{Tr}\left(V\left(\mathbf{b}_{T}\right) V^{\dagger}\left(\mathbf{b}_{T}^{\prime}\right) t^{d} t^{c}\right)\right\rangle\left\langle\left[U\left(\mathbf{x}_{T}\right) U^{\dagger}\left(\mathbf{x}_{T}^{\prime}\right)\right]^{c d}\right\rangle \\
= & \frac{N_{\mathrm{c}}{ }^{2}}{N_{\mathrm{c}}{ }^{2}-1}\left\langle\hat{D}\left(\mathbf{b}_{T}, \mathbf{b}_{T}^{\prime}\right)\right\rangle\left\langle\hat{D}^{2}\left(\mathbf{x}_{T}, \mathbf{x}_{T}^{\prime}\right)-\frac{1}{N_{\mathrm{c}}{ }^{2}}\right\rangle
\end{aligned}
$$

where

$$
\widetilde{S}\left(\mathbf{x}_{T}-\mathbf{x}_{T}^{\prime}\right) \equiv \frac{N_{\mathrm{c}}^{2}}{N_{\mathrm{c}}{ }^{2}-1}\left\langle\hat{D}^{2}\left(\mathbf{x}_{T}, \mathbf{x}_{T}^{\prime}\right)-\frac{1}{N_{\mathrm{c}}{ }^{2}}\right\rangle
$$

can be identified as the two point function in the adjoint representation, normalized to $\widetilde{S}\left(\mathbf{x}_{T}=\mathbf{x}_{T}^{\prime}\right)=1$. The cross terms $S^{(3)}$ vanish in the DPS limit. This is easily seen by noting that $z$ is finite as it is fixed by the final state kinematics and thus all three coordinates $\mathbf{b}_{T}, \mathbf{x}_{T}$ and $\mathbf{z}_{T}^{\prime}$ (or $\mathbf{b}_{T}^{\prime}, \mathbf{x}_{T}^{\prime}$ and $\mathbf{z}_{T}$ ) are much more than $1 / Q_{\mathrm{s}}$ apart. Consequently $S^{(3)}$ factorizes into a product of three single Wilson line expectation values, which are all zero. The Wilson line operator corresponding to gluon emission after the target, $S^{(2)}$, is finite in the DPS limit. It does not, as we show in Appendix A give a divergent contribution to the double inclusive cross section even for massless quarks.

In the massless case the integral (5) diverges logarithmically in the DPS limit, because $S^{(4)}$ is nonzero and the wave function product has a large power law tail at large $\mathbf{u}_{T}, \mathbf{u}_{T}^{\prime}$. This logarithmic divergence is shown explicitly in Appendix A. Physically this means that the quark emits a very small transverse momentum gluon. The quark and gluon subsequently scatter independently off the target. A simple lifetime argument shows that the emission of the quark happens $\Delta x^{+} \sim z(1-z) p^{+} u_{T}^{2}$ before the interaction with the target. Thus the contribution from $u_{T} \rightarrow \infty$ corresponds to a splitting happening a long time before the interaction with the target. This logaritmically divergent contribution must be regulated by confinement scale physics in the wavefunction of the projectile. It is in fact exactly the kind of contribution that is represented by double parton scattering [41, 42. The confinement scale physics of the correlations in the large $x$ projectile is not calculable in our formalism. We shall instead add it as an additional explicit DPS contribution. To avoid double counting we must subtract the equivalent contribution from our Eq. (5), which will render it manifestly IR finite. We do this by replacing $S^{(4)}\left(\mathbf{b}_{T}, \mathbf{x}_{T}, \mathbf{b}_{T}^{\prime}, \mathbf{x}_{T}^{\prime}\right)$ in Eq. (5) by

$$
\begin{aligned}
& S_{\mathrm{sub}}^{(4)}\left(\mathbf{b}_{T}, \mathbf{x}_{T}, \mathbf{b}_{T}^{\prime}, \mathbf{x}_{T}^{\prime}\right)=S^{(4)}\left(\mathbf{b}_{T}, \mathbf{x}_{T}, \mathbf{b}_{T}^{\prime}, \mathbf{x}_{T}^{\prime}\right) \\
& -\theta\left(\left|\mathbf{x}_{T}-\mathbf{b}_{T}\right|-\frac{1}{\Lambda_{\mathrm{QCD}}}\right) \theta\left(\left|\mathbf{x}_{T}^{\prime}-\mathbf{b}_{T}^{\prime}\right|-\frac{1}{\Lambda_{\mathrm{QCD}}}\right) \\
& \times S_{\mathrm{DPS}}^{(4)}\left(\mathbf{b}_{T}, \mathbf{x}_{T}, \mathbf{b}_{T}^{\prime}, \mathbf{x}_{T}^{\prime}\right)
\end{aligned}
$$

with $S_{\text {DPS }}^{(4)}$ given by Eq. 14 . 
After convoluting with the valence quark distribution in the probe deuteron or proton this correlated, $\Delta \varphi$ - dependent part of the double differential yield becomes

$$
\begin{gathered}
\frac{\mathrm{d} N_{\text {sub }}^{h_{a} h_{b} \rightarrow q g X}}{\mathrm{~d} y_{q} \mathrm{~d}^{2} \mathbf{k}_{T} \mathrm{~d} y_{g} \mathrm{~d}^{2} \mathbf{q}_{T}}=\alpha_{\mathrm{s}} C_{\mathrm{F}} x q\left(x, Q^{2}\right)(1-z)\left(1+(1-z)^{2}\right) \frac{1}{S_{\perp}} \int \frac{\mathrm{d}^{2} \mathbf{x}_{T}}{(2 \pi)^{2}} \frac{\mathrm{d}^{2} \mathbf{x}_{T}^{\prime}}{(2 \pi)^{2}} \frac{\mathrm{d}^{2} \mathbf{b}_{T}}{(2 \pi)^{2}} \frac{\mathrm{d}^{2} \mathbf{b}_{T}^{\prime}}{(2 \pi)^{2}} \\
e^{i \mathbf{k}_{T} \cdot\left(\mathbf{x}_{T}^{\prime}-\mathbf{x}_{T}\right)} e^{i \mathbf{q}_{T} \cdot\left(\mathbf{b}_{T}^{\prime}-\mathbf{b}_{T}\right)} 8 \pi^{2} \frac{\left(\mathbf{x}_{T}-\mathbf{b}_{T}\right) \cdot\left(\mathbf{x}_{T}^{\prime}-\mathbf{b}_{T}^{\prime}\right)}{\left(\mathbf{x}_{T}-\mathbf{b}_{T}\right)^{2}\left(\mathbf{x}_{T}^{\prime}-\mathbf{b}_{T}^{\prime}\right)^{2}} \\
\left\{S_{\mathrm{sub}}^{(4)}\left(\mathbf{b}_{T}, \mathbf{x}_{T}, \mathbf{b}_{T}^{\prime}, \mathbf{x}_{T}^{\prime}\right)-S^{(3)}\left(\mathbf{b}_{T}, \mathbf{x}_{T}, \mathbf{z}_{T}^{\prime}\right)-S^{(3)}\left(\mathbf{z}_{T}, \mathbf{x}_{T}^{\prime}, \mathbf{b}_{T}^{\prime}\right)+S^{(2)}\left(\mathbf{z}_{T}, \mathbf{z}_{T}^{\prime}\right)\right\} .
\end{gathered}
$$

Here we have moved from a cross section to a differential yield by dividing with the transverse area of the target $S_{\perp}$. Because the integrand is translationally invariant as a whole, one out of the four integrals over the transverse plane in Eq. (17) is performed trivially, canceling the factor $1 / S_{\perp}$ (see also the discussion on the impact parameter dependence in Appendix B. We assume the Wilson line correlators to be independent of the overall impact parameter; thus the dependence on centrality only comes through the Wilson line correlator, which should correspond to an average one for the desired centrality class.

Now that the DPS contribution has been subtracted from the dihadron correlation, we must include it separately to get an estimate of the $\Delta \varphi$-independent background. The logarithmic divergence in the DPS limit is physically regulated by confinement scale physics, and must be absorbed into a new nonperturbative input describing the probe, namely the double parton distribution function (DPDF). For deuteron-gold collisions we divide the deuteron DPDF into two separate parts. The first one corresponds to taking two partons from the same nucleon in the deuteron, described by a single-nucleon double parton distribution $D_{i j}\left(x_{i}, x_{j}, Q^{2}\right)$. We implement the kinematical constraint $x_{i}+x_{j}<1$ following Ref. [40] by taking

$$
\begin{aligned}
& D_{i j}^{(1)}\left(x_{i}, x_{j}, Q^{2}\right)=\frac{1}{2}\left[f_{i}(\right.\left.x_{i}\right) f_{j}\left(\frac{x_{j}}{1-x_{j}}\right) \\
&\left.+f_{i}\left(\frac{x_{i}}{1-x_{j}}\right) f_{j}\left(x_{j}\right)\right]
\end{aligned}
$$

where $i$ and $j$ denote the parton species $(g, u, d)$. For the deuteron one must sum over the proton and neutron: $D_{i j}^{d}\left(x_{i}, x_{j}, Q^{2}\right)=D_{i j}^{p}\left(x_{i}, x_{j}, Q^{2}\right)+D_{i j}^{n}\left(x_{i}, x_{j}, Q^{2}\right)$ The second contribution involves taking one parton from the neutron and the other one from the proton, which is not bound by the same kinematical constraint. In this case one must sum; not symmetrize; over the different combinations of different partons from different nucleons:

$$
D_{i j}^{(2)}\left(x_{i}, x_{j}, Q^{2}\right)=\left[f_{i}^{p}\left(x_{i}\right) f_{j}^{n}\left(x_{j}\right)+f_{i}^{p}\left(x_{i}\right) f_{j}^{n}\left(x_{j}\right)\right] .
$$

The double parton scattering contribution is the sum of these two

$$
\begin{array}{r}
\frac{\mathrm{d} N_{\mathrm{DPS}}^{h_{a} h_{b} \rightarrow i j X}}{\mathrm{~d} y_{q} \mathrm{~d}^{2} \mathbf{k}_{T} \mathrm{~d} y_{g} \mathrm{~d}^{2} \mathbf{q}_{T}}=\frac{1}{(2 \pi)^{4}}\left(D_{i j}^{(1)}\left(x_{i}, x_{j}, Q^{2}\right)\right. \\
\left.+D_{i j}^{(2)}\left(x_{i}, x_{j}, Q^{2}\right)\right) S_{i}\left(\mathbf{k}_{T i}\right) S_{j}\left(\mathbf{k}_{T j}\right),
\end{array}
$$

where the two point functions $S_{i}\left(\mathbf{k}_{T i}\right), S_{j}\left(\mathbf{k}_{T j}\right)$ are taken in the fundamental or adjoint representation according to the parton species $i, j$.

The total quark-gluon production cross section is now the sum of 20 and $(17)$ :

$$
\mathrm{d} N^{h_{a} h_{b} \rightarrow i j X}=\mathrm{d} N_{\text {DPS }}^{h_{a} h_{b} \rightarrow i j X}+\mathrm{d} N_{\text {sub }}^{h_{a} h_{b} \rightarrow i j X} .
$$

After convolution with fragmentation functions the cross section for double inclusive production of hadrons 1 and 2 becomes

$$
\begin{aligned}
& \frac{\mathrm{d} N^{h_{a} h_{b} \rightarrow h_{1} h_{2} X}}{\mathrm{~d} y_{1} \mathrm{~d}^{2} \mathbf{p}_{T 1} \mathrm{~d} y_{2} \mathrm{~d}^{2} \mathbf{p}_{T 2}}=\int \frac{\mathrm{d} z_{1}}{z_{1}^{2}} \frac{\mathrm{d} z_{2}}{z_{2}^{2}} \\
& \frac{\mathrm{d} N^{h_{a} h_{b} \rightarrow i j X}}{\mathrm{~d} y_{1} \mathrm{~d}^{2}\left(\mathbf{p}_{T 1} / z_{1}\right) \mathrm{d} y_{2} \mathrm{~d}^{2}\left(\mathbf{p}_{T 2} / z_{2}\right)} D_{h_{1}, i}\left(z_{1}\right) D_{h_{2}, j}\left(z_{2}\right)
\end{aligned}
$$

where $i$ and $j$ are summed over gluons and light quark flavors; for the combination $i, j=q, g$ and $i, j=g, q$ the parton level cross section includes the correlated cross section $\mathrm{d} N_{\mathrm{sub}}^{h_{a} h_{b} \rightarrow i j X}$ and for the others only the DPS contribution. This is our final expression that will be compared to the experimental yield. We shall now turn to the calculation of the different Wilson line correlators appearing in $\mathrm{d} N_{\mathrm{sub}}^{h_{a} h_{b} \rightarrow i j X}$.

\section{MULTI-POINT CORRELATORS OF WILSON LINES}

As pointed out in [15 17, one expects the true JIMWLK result for higher-point correlators of Wilson lines to significantly deviate from the simple product of dipole correlators (called "naive large $N_{\mathrm{c}}$ limit" in [17) 
used in the phenomenological applications so far [23, 43]. It was conjectured in Ref. [17, based on numerical tests for particular coordinate configurations, that JIMWLK evolution for the higher-point correlators of Wilson lines can be approximated by a "Gaussian approximation". Here one constructs, as in the MV model, the Wilson lines from color charge densities whose probability distribution is assumed to be local in rapidity and Gaussian. This enables one to express all higher-point functions in terms of the two point function. The equations relating different Wilson line correlators are the same in the Gaussian approximation of JIMWLK and the MV model, the difference between the two being the different functional form of the two point function (dipole). A theoretical explanation for the success of the Gaussian approximation is given in Ref. [18, 19.

The cross section formula (5) involves correlators of up to six Wilson lines. The general Gaussian approximation for the six point function $S^{(4)}$ is not known at finite $N_{\mathrm{c}}$, so we will here use the large $N_{\mathrm{c}}$ limit. We shall also compare the large $N_{\mathrm{c}}$ limit to the approach used in Ref. [17] for the six point function, which we call here the "factorized Gaussian" approximation. This consists of expressing all the higher-point functions as products of fundamental representation traces, and then assuming that the expectation value of a product of traces factorizes into a product of expectation values. While this factorization would follow from the large $N_{\mathrm{c}}$-limit, it is a consistent approximation scheme in itself also at finite $N_{\text {c }}$. The "factorized Gaussian" has the advantage of preserving all the "coincidence limits", i.e. it correctly incorporates the constraints coming from the $\mathrm{SU}\left(N_{\mathrm{c}}\right)$ group definition $V\left(\mathbf{x}_{T}\right) V^{\dagger}\left(\mathbf{y}_{T}\right) \rightarrow 1$, when $\mathbf{x}_{T} \rightarrow \mathbf{y}_{T}$. At a minimum, comparing this approximation to the large- $N_{\mathrm{c}}$ result should give us an indication of the size of finite $N_{\text {c }}$ effects. Evidence from a numerical calculation [44] of two traces appearing on the r.h.s. of the BK equation shows that, at least in this particular case, the "factorized Gaussian" approximation is much more accurate than the $10 \%$ level suggested by simple $N_{\mathrm{c}}$ counting. While for the four-point function $S^{(3)}$ it would be possible to use the full Gaussian approximation, we will, for consistency, use the same approach also for $S^{(3)}$. Thus the "factorized Gaussian" formulae for the Wilson line correlators used in Eq. (5) are

$$
\begin{aligned}
S^{(4)}\left(\mathbf{b}_{T}, \mathbf{b}_{T}^{\prime}, \mathbf{x}_{T}, \mathbf{x}_{T}^{\prime}\right) & \approx \frac{N_{\mathrm{c}}^{2}}{N_{\mathrm{c}}^{2}-1}\left[S\left(\mathbf{x}_{T}, \mathbf{x}_{T}^{\prime}\right) Q\left(\mathbf{b}_{T}, \mathbf{b}_{T}^{\prime}, \mathbf{x}_{T}, \mathbf{x}_{T}^{\prime}\right)-\frac{1}{N_{\mathrm{c}}^{2}} S\left(\mathbf{b}_{T}, \mathbf{b}_{T}^{\prime}\right)\right] \\
S_{\mathrm{DPS}}^{(4)}\left(\mathbf{b}_{T}, \mathbf{b}_{T}^{\prime}, \mathbf{x}_{T}, \mathbf{x}_{T}^{\prime}\right) & \approx \frac{N_{\mathrm{c}}^{2}}{N_{\mathrm{c}}^{2}-1} S\left(\mathbf{b}_{T}, \mathbf{b}_{T}^{\prime}\right)\left[S\left(\mathbf{x}_{T}, \mathbf{x}_{T}^{\prime}\right)^{2}-\frac{1}{N_{\mathrm{c}}^{2}}\right] \\
S^{(3)}\left(\mathbf{b}_{T}, \mathbf{x}_{T}, \mathbf{z}_{T}^{\prime}\right) & \approx \frac{N_{\mathrm{c}}^{2}}{N_{\mathrm{c}}^{2}-1}\left[S\left(\mathbf{b}_{T}, \mathbf{x}_{T}\right) S\left(\mathbf{x}_{T}, \mathbf{z}_{T}^{\prime}\right)-\frac{1}{N_{\mathrm{c}}^{2}} S\left(\mathbf{b}_{T}, \mathbf{z}_{T}^{\prime}\right)\right]
\end{aligned}
$$

where we use the exact Gaussian quadrupole $Q\left(\mathbf{b}_{T}, \mathbf{b}_{T}^{\prime}, \mathbf{x}_{T}, \mathbf{x}_{T}^{\prime}\right)$ calculated in Ref. [24]; since its expression is rather cumbersome we will not repeat it here.

In the large $N_{\mathrm{c}}$ limit the Gaussian approximation reduces to

$$
\begin{aligned}
S^{(4)}\left(\mathbf{b}_{T}, \mathbf{b}_{T}^{\prime}, \mathbf{x}_{T}, \mathbf{x}_{T}^{\prime}\right) \underset{N_{\mathrm{c}} \rightarrow \infty}{\approx} S\left(\mathbf{x}_{T}, \mathbf{x}_{T}^{\prime}\right)\left[S\left(\mathbf{b}_{T}-\mathbf{x}_{T}\right) S\left(\mathbf{x}_{T}^{\prime}-\mathbf{b}_{T}^{\prime}\right)\right. \\
\\
\left.-\frac{F\left(\mathbf{b}_{T}, \mathbf{x}_{T}, \mathbf{x}_{T}^{\prime}, \mathbf{b}_{T}^{\prime}\right)}{F\left(\mathbf{b}_{T}, \mathbf{x}_{T}^{\prime}, \mathbf{x}_{T}, \mathbf{b}_{T}^{\prime}\right)}\left(S\left(\mathbf{b}_{T}-\mathbf{x}_{T}\right) S\left(\mathbf{x}_{T}^{\prime}-\mathbf{b}_{T}^{\prime}\right)-S\left(\mathbf{b}_{T}-\mathbf{b}_{T}^{\prime}\right) S\left(\mathbf{x}_{T}^{\prime}-\mathbf{x}_{T}\right)\right)\right], \\
S_{\mathrm{DPS}}^{(4)}\left(\mathbf{b}_{T}, \mathbf{b}_{T}^{\prime}, \mathbf{x}_{T}, \mathbf{x}_{T}^{\prime}\right) \underset{N_{\mathrm{c}} \rightarrow \infty}{\approx} S\left(\mathbf{b}_{T}, \mathbf{b}_{T}^{\prime}\right) S\left(\mathbf{x}_{T}, \mathbf{x}_{T}^{\prime}\right)^{2} \\
S^{(3)}\left(\mathbf{b}_{T}, \mathbf{x}_{T}, \mathbf{z}_{T}^{\prime}\right) \underset{N_{\mathrm{c}} \rightarrow \infty}{\approx} S\left(\mathbf{b}_{T}, \mathbf{x}_{T}\right) S\left(\mathbf{x}_{T}, \mathbf{z}_{T}^{\prime}\right)
\end{aligned}
$$

with the auxiliary function

$$
\frac{F\left(\mathbf{b}_{T}, \mathbf{x}_{T}, \mathbf{x}_{T}^{\prime}, \mathbf{b}_{T}^{\prime}\right)}{F\left(\mathbf{b}_{T}, \mathbf{x}_{T}^{\prime}, \mathbf{x}_{T}, \mathbf{b}_{T}^{\prime}\right)}=\frac{\ln S\left(\mathbf{b}_{T}, \mathbf{x}_{T}^{\prime}\right)-\ln S\left(\mathbf{b}_{T}, \mathbf{b}_{T}^{\prime}\right)+\ln S\left(\mathbf{x}_{T}, \mathbf{b}_{T}^{\prime}\right)-\ln S\left(\mathbf{x}_{T}, \mathbf{x}_{T}^{\prime}\right)}{\ln S\left(\mathbf{b}_{T}, \mathbf{x}_{T}\right)-\ln S\left(\mathbf{b}_{T}, \mathbf{b}_{T}^{\prime}\right)+\ln S\left(\mathbf{x}_{T}^{\prime}, \mathbf{b}_{T}^{\prime}\right)-\ln S\left(\mathbf{x}_{T}^{\prime}, \mathbf{x}_{T}\right)}
$$

The first term in Eq. 26 is the "elastic" term, which is the only one kept in Ref. [22]. The second term is some- 


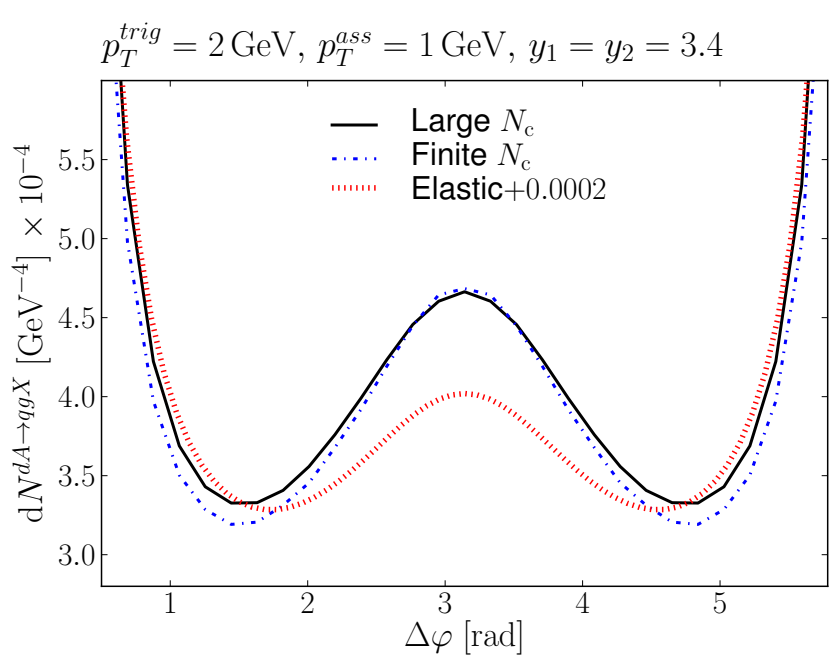

FIG. 3: The quark-gluon parton level azimuthal correlation in dAu collisions, Eq. (17), near forward RHIC kinematics, at transverse momenta $p_{T}^{\text {trrg }}=2 \mathrm{GeV}, p_{T}^{\text {ass }}=1 \mathrm{GeV}$ and $y=$ 3.4. Shown are the "naive large $N_{\mathrm{c}}$ " approximation with only the "elastic" contribution used in Ref. 23. and our Gaussian approximation of Eq. (23) and its large $N_{\mathrm{c}}$ limit Eq. (26). Note that a fixed $\Delta \varphi$-independent pedestal of $0.0002 \mathrm{GeV}^{-4}$ has been added to the "elastic" approximation for purposes of visualization. The DPS contribution is not included here.

times referred to as the "inelastic" one. Note that one can explicitly verify that the limiting behavior $S^{(4)} \rightarrow S_{\mathrm{DPS}}^{(4)}$ holds both for the factorized Gaussian approximation (23) and its large $N_{\mathrm{c}}$ limit (26). The nonzero contribution in the DPS limit comes only from the "inelastic" part of $S^{(4)}$ which was neglected in Ref. [22]; thus the logarithmic divergence in this limit did not appear in that calculation.

\section{RESULTS}

We begin this section by showing results at the parton level, demonstrating the systematics as a function of different trigger and associate momenta, different probe and target species. We then include the fragmentation functions for a more realistic comparison with both STAR and PHENIX dipion correlations.

Figure 3 shows the effect of the "inelastic" contribution neglected in Ref. 23] to the dihadron correlation. Keeping only the "elastic" term, as done in Ref. 223, results in an away side peak that is smaller by a factor of $\sim 2$ than the factorized Gaussian approximation, while the effect on the width of the peak is much smaller. Our conclusion from this plot is that including both contributions is essential for a meaningful comparison with experimental data. We also notice that the finite $N_{\mathrm{c}}$ corrections to the Gaussian approximation can be neglected, and from now on we only use the large $N_{\mathrm{c}}$ version of the Gaussian approximation. The initial saturation scale for the nucleus
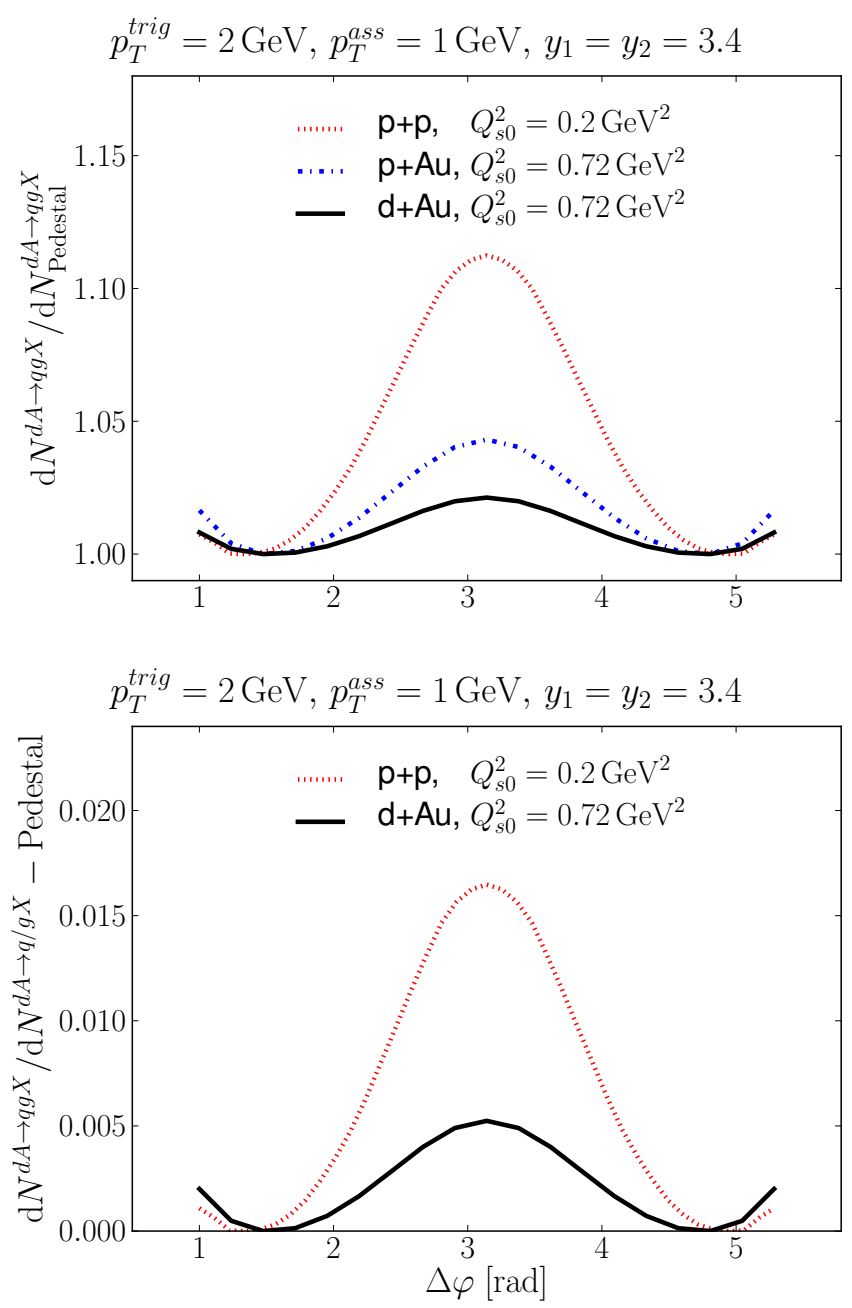

FIG. 4: The quark-gluon parton level azimuthal correlation at forward RHIC kinematics in proton-proton, protonnucleus and deuteron-nucleus collisions. Top: normalized by the pedestal contribution, bottom: pedestal contribution subtracted (in which case the $\mathrm{pAu}$ and $\mathrm{dAu}$ results are identical by construction). Shown is only the large $N_{\mathrm{c}}$ result.

is taken as $Q_{\mathrm{s} 0}^{2}=0.72 \mathrm{GeV}^{2}$.

Figure 4 illustrates the difference between deuteronnucleus, proton-nucleus and proton-proton collisions in the ratio of the peak to the $\Delta \varphi$-independent background. Plotted on top is the total dihadron cross section, including the DPS contribution as discussed in Sec. IV] divided by the minimum of the $\Delta \varphi$-distribution. On the bottom this pedestal is subtracted. The initial saturation scales are taken as $Q_{\mathrm{s} 0}=0.2 \mathrm{GeV}^{2}$ for the proton and $Q_{\mathrm{s} 0}^{2}=0.72 \mathrm{GeV}^{2}$ for a nucleus. Comparing the pp and $\mathrm{pA} / \mathrm{dA}$ results one sees clearly the depletion of the peak relative to pp collisions when the target is a large nucleus. This is the crucial generic feature whose observation in the experimental data supports the concept of a depletion caused by intrinsic gluon transverse momenta 


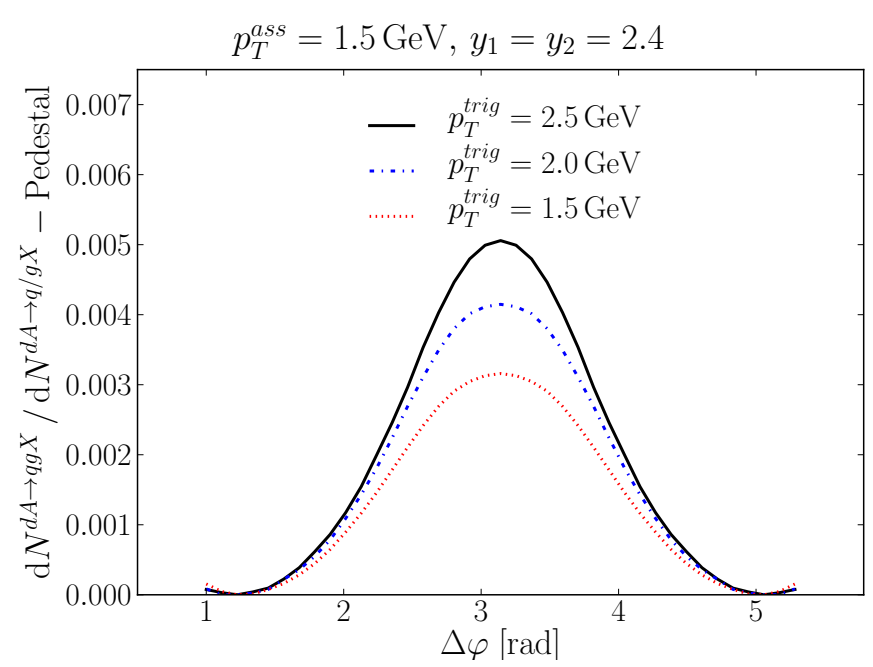

FIG. 5: The pedestal-subtracted quark-gluon parton level azimuthal correlation at forward RHIC kinematics, at different values of the trigger transverse momenta $p_{T}^{\text {trig }}=$ $1.5,2,2.5 \mathrm{GeV}$ and $y=2.4$. Shown is only the large $N_{\mathrm{c}}$ result.

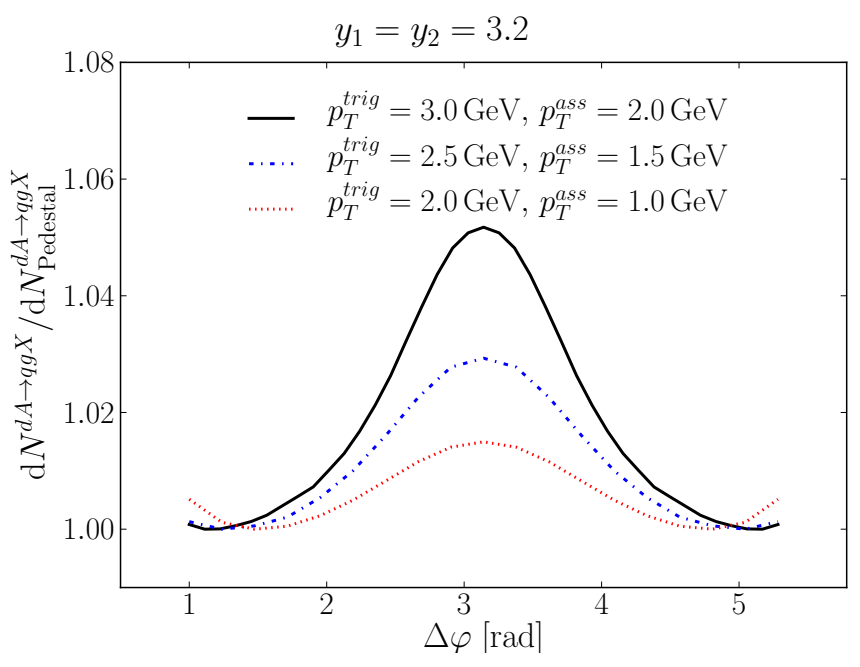

FIG. 6: The total quark-gluon parton level dihadron production yield at forward RHIC kinematics at different values of the trigger and associated particle transverse momenta at $y=3.2$. The DPS contribution is included and the result is normalized by the pedestal yield. Shown is only the large $N_{\mathrm{c}}$ result.

in the target given by a saturation scale. Since we are neglecting any differences in the large $x$ quark distribution between a deuteron and a proton, there is no difference in the correlated peak between $\mathrm{pAu}$ and $\mathrm{dAu}$ collisions. Due to the enhanced DPS contribution in deuteron collisions (where the possibility of taking one large $x$ quark from the proton and the other from the neutron makes it much easier to observe a double scattering event in very forward kinematics), the DPS contribution is much

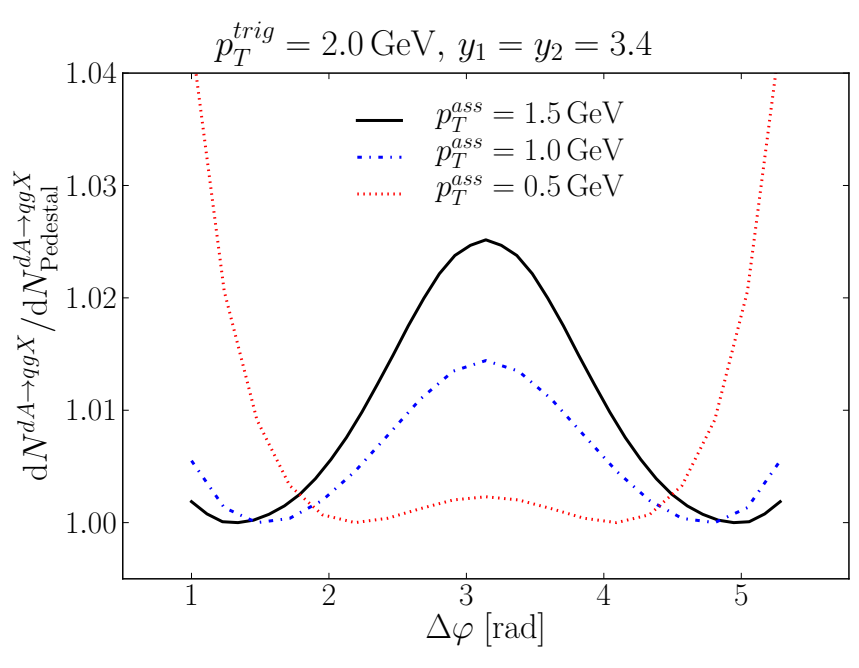

FIG. 7: The total quark-gluon parton level dihadron production yield at forward RHIC kinematics, for trigger transverse momentum $p_{T}^{\text {trig }}=2 \mathrm{GeV}$, and different values of the associate transverse momentum $p_{T}^{\text {ass }}=0.5,1,1.5 \mathrm{GeV}$ and $y=3.4$. Shown is only the large $N_{\mathrm{c}}$ result. The DPS contribution is included and the result is normalized by the pedestal yield.

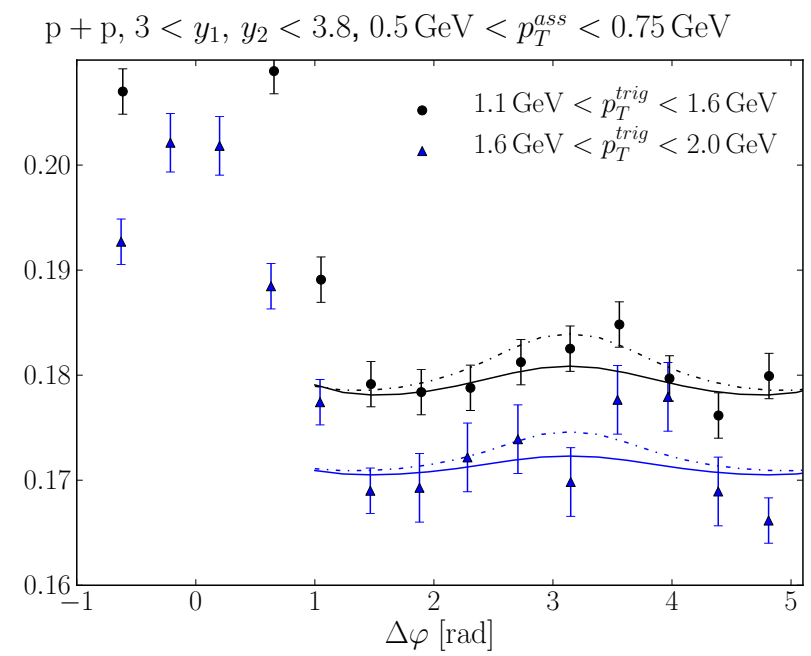

FIG. 8: The $\pi^{0}$ azimuthal correlation compared to the PHENIX [1] dAu result for two different $p_{T}^{\text {trig }}$ bins. The $\Delta \varphi$-independent pedestal in the plot is adjusted to fit the ex-

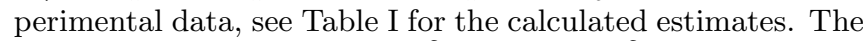
initial saturation scales are $Q_{\mathrm{s} 0}^{2}=1.51 \mathrm{GeV}^{2}$ (solid line) and $Q_{\mathrm{s} 0}^{2}=0.72 \mathrm{GeV}^{2}$ (dashed line).

larger in $\mathrm{dAu}$ collisions. This leads to a smaller ratio of the correlated peak to the pedestal, purely because of the increase in the denominator. The effect is similar to what is seen in the STAR forward neutron tagged (effectively pAu) data [45] and discussed in Ref. 40].

Figure 5 shows the dependence on the parton level $\mathrm{dAu}$ result on the trigger transverse momentum. Shown 


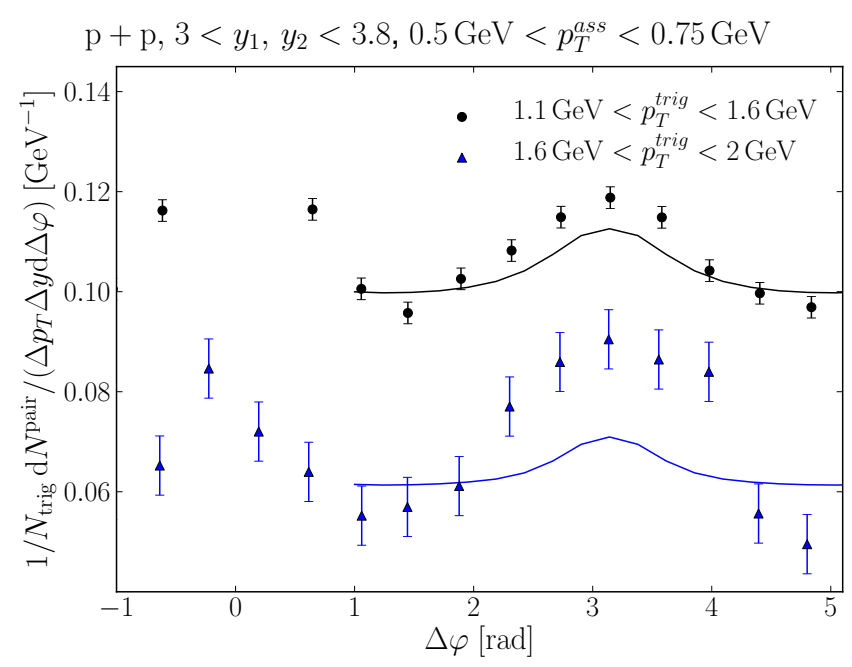

FIG. 9: The $\pi^{0}$ azimuthal correlation compared to the PHENIX 11] pp result, for the same transverse momenta as in Fig. 8 The $\Delta \varphi$-independent pedestal in the plot is adjusted to fit the experimental data, see Table I for the calculated estimates. The initial condition is the MV model with initial saturation scale $Q_{\mathrm{s} 0}^{2}=0.2 \mathrm{GeV}^{2}$. Data points at larger $p_{T}^{\text {trig }}$ are sifted by $-0.03 \mathrm{GeV}^{-1}$ to avoid overlap.

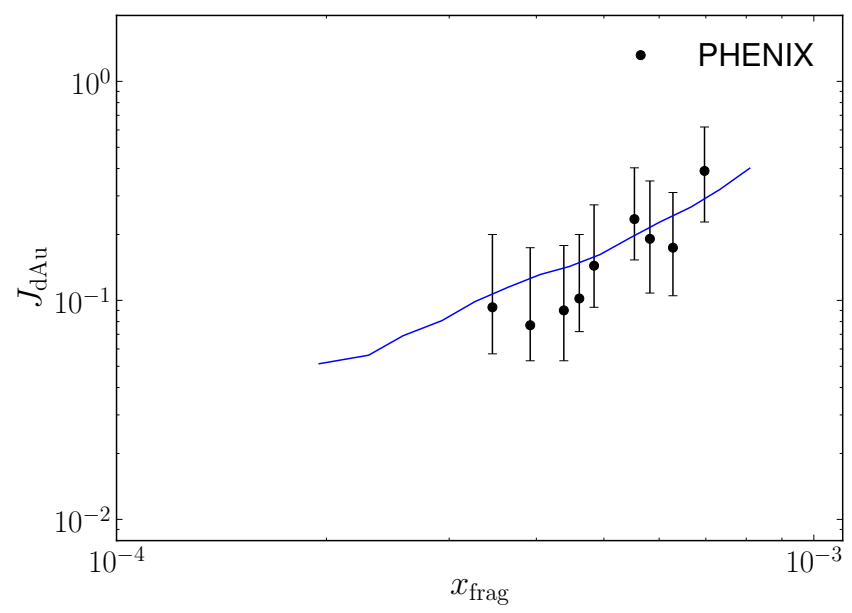

FIG. 10: Integrated yield under the away side peak in central $\mathrm{dAu}$ collision divided by the corresponding yield in pp compared to the forward rapidity part of the PHENIX data [1].

is the pedestal-subtracted yield per trigger for associate parton transverse momentum $p_{T}=1.5 \mathrm{GeV}$ and trigger transverse momenta $p_{T}=1.5,2.0,2.5 \mathrm{GeV}$. These should be compared to the typical nuclear saturation scale $Q_{\mathrm{s}} \approx 1.5 \mathrm{GeV}$ at these rapidities (defining $Q_{\mathrm{s}}$ via the saturation criterion $\left.S\left(r=1 / Q_{\mathrm{s}}\right)=1-e^{-1 / 4}\right)$. One again sees clearly the expected effect of the nuclear saturation scale: the peak is small for small trigger transverse momentum, but starts to reappear when the trigger

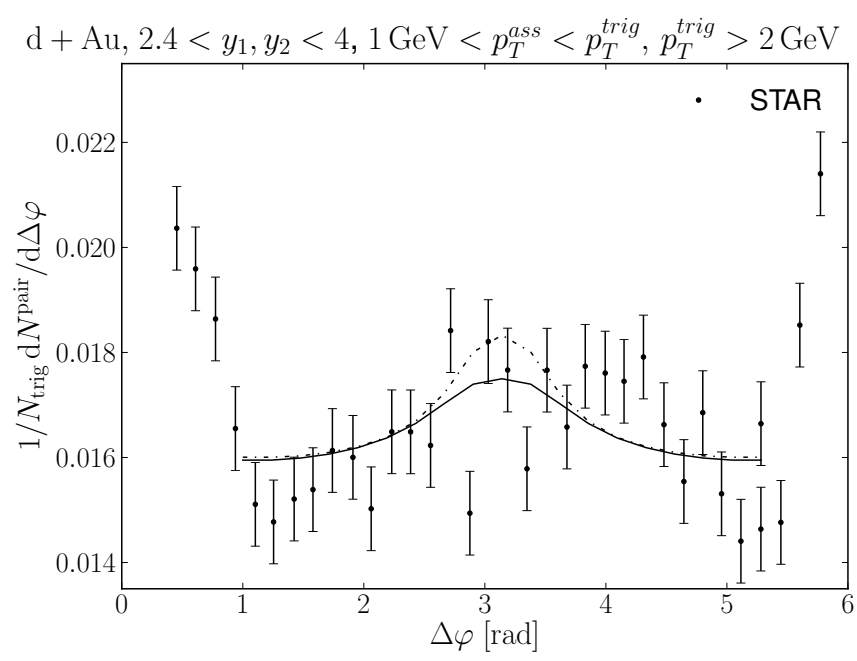

FIG. 11: The $\pi^{0}$ azimuthal correlation compared to the preliminary STAR 10 result. The initial saturation scales are $Q_{\mathrm{s} 0}^{2}=1.51 \mathrm{GeV}^{2}$ (solid line) and $Q_{\mathrm{s} 0}^{2}=0.72 \mathrm{GeV}^{2}$ (dashed line).

momentum is increased. Note that the rapidity in this plot is slightly less forward than elsewhere. This is to avoid hitting the kinematical limit $x_{g}+x_{q}<1$ at large $x$ in the deuteron. At very forward rapidites and high enough transverse momenta the kinematical limit causes the height of the peak in the yield per trigger to decrease when the trigger $p_{T}$ is increased. This is caused by the fact that the correlated quark-gluon pair in the numerator of the ratio requires a larger total longitudinal momentum (and is thus suppressed at $x \rightarrow 1$ ) than the single quark (at the same $y$ and $p_{T}$ as the trigger) in the single inclusive cross section in the denominator. Because of this effect the dependence of the peak height on the trigger $p_{T}$ at forward RHIC kinematics is actually smaller than the ratio of the momentum to the saturation scale would suggest.

Figures 6 and 7 show the effect on the yield per trigger of varying also the associate $p_{T}$. Since the two particle yield (just like the single inclusive associate particle yield) is a steeply falling function of the associate $p_{T}$, the yield per trigger itself falls as a function of $p_{T}^{\text {ass }}$. To show what is actually happening to the peak with respect to the background we again include the DPS contribution to the pedestal and divide the correlation by the $\Delta \varphi$ independent part. As expected, the away-side peak is strongly suppressed for smaller trigger $p_{T}$.

Figures 8 and 9 compare our calculation of the away side correlation to the PHENIX [11] data for different momentum bins. Figure 9 shows the proton-proton and 8 the central deuteron-gold result. For the latter we show calculations using two initial values for the saturation scale: $Q_{\mathrm{s} 0}^{2}=1.51 \mathrm{GeV}^{2}$, which would be the natural estimate for these central collisions, and a smaller value $Q_{\mathrm{s}}^{2}=0.72 \mathrm{GeV}^{2}$, which would be preferred by some of 


\begin{tabular}{|l|l|r|r|}
\hline Data & $p_{T}$ range & pedestal & exp. \\
\hline \hline PHENIX pp & $1.1 \mathrm{GeV}<p_{T}^{\text {trig }}<1.6 \mathrm{GeV}$ & 0.04 & 0.095 \\
\hline PHENIX pp & $1.6 \mathrm{GeV}<p_{T}^{\text {trig }}<2.0 \mathrm{GeV}$ & 0.02 & 0.079 \\
\hline PHENIX dAu & $1.1 \mathrm{GeV}<p_{T}^{\text {trig }}<1.6 \mathrm{GeV}$ & 0.10 & 0.176 \\
\hline PHENIX dAu & $1.6 \mathrm{GeV}<p_{T}^{\text {trig }}<2.0 \mathrm{GeV}$ & 0.08 & 0.163 \\
\hline STAR dAu & $2 \mathrm{GeV}<p_{T}^{\text {trig }}, 1 \mathrm{GeV}<p_{T}^{\text {ass }}<p_{T}^{\text {trig }}$ & 0.02 & 0.0145 \\
\hline
\end{tabular}

TABLE I: Calculated estimates for the pedestal height compared to the experimental values. In PHENIX results the units are $\mathrm{GeV}^{-1}$. The $\mathrm{dAu}$ values are for central collisions, and $Q_{\mathrm{s} 0}^{2}$ is taken as $Q_{\mathrm{s} 0}^{2}=1.51 \mathrm{GeV}^{2}$.

the single inclusive data.

Due to the significant uncertainty in the single inclusive baseline spectrum, the $\Delta \varphi$-independent pedestal values are rough estimates that cannot be directly compared to the data at this point. For plotting purposes the pedestal values have been adjusted to the data in the plot; the results of the calculation compared to the experimental pedestal are given in Table II The normalization uncertainty also affects the height of the away side peak, even though we would expect that at least part of this theoretical uncertainty cancels in the yield-pertrigger ratio. Taking into account these uncertainties, we obtain relatively good description of the back-to-back peak, underestimating the peak height especially with $Q_{\mathrm{s} 0}^{2}=1.51 \mathrm{GeV}^{2}$ for the nucleus and in the case of the proton-proton scattering.

PHENIX has also published data for larger trigger particle momentum $2<p_{T}^{\text {trig }}<5 \mathrm{GeV}$. We can not describe the observed relative increase of the peak height compared to lower values of the trigger momenta in this kinematics. This phenomenon can be seen in parton level results (see Fig. 5), but in PHENIX kinematics we are so close to the kinematical boundary that in our calculation the deuteron parton distribution function suppresses the peak.

Figure 10 compares our result for the nuclear modification factor $J_{\mathrm{dAu}}$ of the area under the peak to the PHENIX experimental result [11. The experimental data are given as a function of $x_{\text {frag }} \equiv$ $\left(\left\langle p_{T}^{\text {trig }}\right\rangle e^{-\left\langle\eta_{\text {trig }}\right\rangle}+\left\langle p_{T}^{\text {ass }}\right\rangle e^{-\left\langle\eta_{\text {ass }}\right\rangle}\right) / \sqrt{s_{N N}}$. We compute this quantity by calculating the ratios of the areas under the pp and $\mathrm{dAu}$ away side peaks at various kinematical configurations at rapidities $3<y_{1}, y_{2}<3.8$ and at various trigger $\left(1<p_{T}^{\text {trig }}<2 \mathrm{GeV}\right)$ and associate particle $\left(0.5<p_{T}^{\text {ass }}<1.5 \mathrm{GeV}\right)$ transverse momenta. We then obtain $J_{\mathrm{dAu}}$ by averaging the results in every $x_{\text {frag }}$ bin. This is not exactly the same method that is used in experiments, but we have tested that the result does not depend much on the kinematical limits. Our calculation agrees with the PHENIX result within errors, and we clearly see that the suppression increases when $x_{\text {frag }}$ decreases, which is expected as the saturation effects should become more visible at smaller $x_{\text {frag }}$. The peak height is underestimated in both pp and $\mathrm{dAu}$ collisions probably due to the uncertainties in the single inclusive baseline normalization, and we expect that this error partly can- cels in $J_{\mathrm{dAu}}$.

Finally Fig. 11 compares our calculation to the STAR [10] result. While the errors in the data are rather large, let us point out a few things. Our calculation seems to agree reasonably well with the data, although perhaps underestimating the hight of the peak. It is clear from Fig. 3 that neglecting the "inelastic" part of the cross section (as done by Albacete and Marquet 23]) would make the agreement worse. ${ }^{2}$ Note also that we are assuming collinear factorization of partons into hadrons, which is very crude at these small transverse momenta. Any $k_{T}$-smearing from fragmentation would broaden the away-side peak.

\section{CONCLUSIONS}

We have in this paper performed a calculation of nuclear modifications of forward dihadron correlations in the CGC framework. We use a running coupling BK evolution for the dipole and a factorized Gaussian approximation for the higher-point functions of Wilson lines to describe the target. Our calculation is the first one in the literature to evaluate the the full expression of the dihadron cross section, keeping both the "elastic" and "inelastic" terms (unlike Ref. [23]) and not restricted to the high- $p_{T}$ "correlation limit" (unlike Ref. 25]). We find that including the "inelastic" term in has a significant effect on the correlation, enhancing the peak by a factor $\sim 2$. We also show that in the appropriate kinematics the dihadron cross section reduces to $\Delta \varphi$-independent double parton scattering, which in the earlier literature has been considered as a completely separate contribution.

We show explicitly how the large saturation scale in a nucleus leads to a smoothing of the away-side peak in dihadron correlations for transverse momenta of the order of the saturation scale. We obtain a reasonable order-of-magnitude estimate also for the $\Delta \varphi$-independent pedestal part of the correlation. Taking into account the lack of a parametrization of the dipole cross section

\footnotetext{
2 When comparing to the result in Ref. 23] note also that there is a significant numerical error in the calculation of Ref. 23], which we thank C. Marquet for indicating to us.
} 


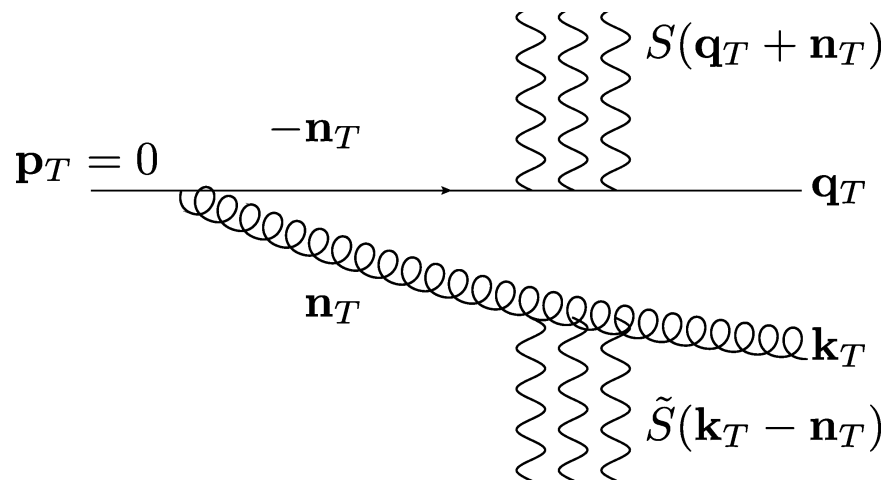

FIG. 12: Illustration of the kinematics in Eq. A5.

that would simultaneously reproduce both mid- and forward rapidity single inclusive hadron spectra from all the RHIC experiments (including the STAR and PHENIX forward $\pi^{0}$ data sets for which the correlation measurements are performed) it is difficult to consistently perform a more accurate calculation of the pedestal contribution at this point. We thus leave a more thorough exploration of the single inclusive spectra for future work. Results from the future LHC proton-nucleus run, with the significantly larger kinematical coverage available, should significantly clarify these uncertainties. A realistic treatment of the LHC kinematics will require taking into account also the gluon-initiated channel, which has not been done yet in this work.

\section{Acknowledgements}

We thank K. J. Eskola, I. Helenius, R. Paatelainen, B. Schenke, M. Strikman and R. Venugopalan for discussions and J. Albacete and C. Marquet for helpful comparisons with their results. H.M. is supported by the Graduate School of Particle and Nuclear Physics. This work has been supported by the Academy of Finland, projects 141555 and 133005, and by computing resources from CSC - IT Center for Science in Espoo, Finland.

\section{Appendix A: $S^{(4)}$ and $S^{(2)}$ in the DPS limit}

Let us now show that only the four point function $S^{(4)}$ gives an infrared divergent contribution to the the double inclusive cross section. We then show how this contribution can be identified as the double parton scattering contribution appearing in the literature, although calculated in the "hybrid formalism" and not collinearly factorized perturbation theory as usual.

In the DPS limit and for massless quarks the double inclusive cross section Eq. (5) reduces to

$$
\begin{array}{r}
\frac{\mathrm{d} \sigma^{h A \rightarrow q g X}}{\mathrm{~d} y_{q} \mathrm{~d} y_{g} \mathrm{~d}^{2} \mathbf{q}_{T} \mathrm{~d}^{2} \mathbf{k}_{T}} \underset{D P}{=} x q_{h}(x) z(1-z) \alpha_{\mathrm{s}} \int \frac{\mathrm{d}^{2} \mathbf{x}_{T}}{(2 \pi)^{2}} \frac{\mathrm{d}^{2} \mathbf{x}_{T}^{\prime}}{(2 \pi)^{2}} \frac{\mathrm{d}^{2} \mathbf{b}_{T}}{(2 \pi)^{2}} \frac{\mathrm{d}^{2} \mathbf{b}_{T}^{\prime}}{(2 \pi)^{2}} e^{i \mathbf{k}_{T} \cdot\left(\mathbf{x}_{T}^{\prime}-\mathbf{x}_{T}\right)} e^{i \mathbf{q}_{T} \cdot\left(\mathbf{b}_{T}^{\prime}-\mathbf{b}_{T}\right)} 8 \pi^{2} \frac{\mathbf{u}_{T} \cdot \mathbf{u}_{T}^{\prime}}{\left|\mathbf{u}_{T}\right|^{2}\left|\mathbf{u}_{T}^{\prime}\right|^{2}} \\
P_{q \rightarrow q g}(z)\left[S\left(\mathbf{b}_{T}-\mathbf{b}_{T}^{\prime}\right) \widetilde{S}\left(\mathbf{x}_{T}-\mathbf{x}_{T}^{\prime}\right)+S\left(z\left(\mathbf{x}_{T}-\mathbf{x}_{T}^{\prime}\right)+(1-z)\left(\mathbf{b}_{T}-\mathbf{b}_{T}^{\prime}\right)\right)\right],
\end{array}
$$

where we recall that $\widetilde{S}$ denotes the adjoint representation two point function and that $\mathbf{u}_{T}=\mathbf{x}_{T}-\mathbf{b}_{T} ; \mathbf{u}_{T}^{\prime}=\mathbf{x}_{T}^{\prime}-$ $\mathbf{b}_{T}^{\prime}$. We have denoted the splitting function for radiating a gluon from a quark by $P_{q \rightarrow q g}(z)=C_{\mathrm{F}}\left(1+(1-z)^{2}\right) / z$. Using the momentum representation

$$
2 \pi \frac{\mathbf{u}_{T}}{\mathbf{u}_{T}^{2}}=-i \int \mathrm{d}^{2} \mathbf{n}_{T} e^{i \mathbf{n}_{T} \cdot \mathbf{u}_{T}} \frac{\mathbf{n}_{T}}{\mathbf{n}_{T}^{2}},
$$

shifting the integration variables $\mathbf{x}_{T}$ and $\mathbf{b}_{T}$ by $\mathbf{x}_{T}^{\prime}$ and $\mathbf{b}_{T}^{\prime}$ respectively, then integrating over $\mathbf{x}_{T}^{\prime}$ and $\mathbf{b}_{T}^{\prime}$ we get

$$
\begin{array}{r}
\frac{\mathrm{d} \sigma^{h A \rightarrow q g X}}{\mathrm{~d} y_{q} \mathrm{~d} y_{g} \mathrm{~d}^{2} \mathbf{q}_{T} \mathrm{~d}^{2} \mathbf{k}_{T}} \underset{D \bar{P} S}{=} x q_{h}(x) z(1-z) \alpha_{\mathrm{S}} S_{\perp} \frac{1}{(2 \pi)^{6}} \int \mathrm{d}^{2} \mathbf{x}_{T} \mathrm{~d}^{2} \mathbf{b}_{T} \frac{\mathrm{d}^{2} \mathbf{n}_{T}}{\mathbf{n}_{T}^{2}} 2 e^{-i\left(\mathbf{k}_{T}-\mathbf{n}_{T}\right) \cdot \mathbf{x}_{T}} e^{-i\left(\mathbf{q}_{T}+\mathbf{n}_{T}\right) \cdot \mathbf{b}_{T}} \\
P_{q \rightarrow q g}(z)\left[S\left(\mathbf{b}_{T}\right) \widetilde{S}\left(\mathbf{x}_{T}\right)+S\left(z \mathbf{x}_{T}+(1-z) \mathbf{b}_{T}\right)\right]
\end{array}
$$

Let us first look at the second term, which originates

in $S^{(2)}$. Changing the integration variable from $\mathbf{b}_{T}$ to 
$\mathbf{z}_{T}=z \mathbf{x}_{T}+(1-z) \mathbf{b}_{T}$ the $\mathbf{z}_{T}$-integral gives a two-point function in momentum space and the $\mathbf{x}_{T}$-integral a $\delta$ function which can be used to integrate over $\mathbf{n}_{T}$ to get

$$
x q_{h}(x) z(1-z) \alpha_{\mathrm{s}} S_{\perp} \frac{1}{(2 \pi)^{4}} 2 P_{q \rightarrow q g}(z) \frac{S\left(\mathbf{q}_{T}+\mathbf{k}_{T}\right)}{\left[(1-z) \mathbf{k}_{T}-z \mathbf{q}_{T}\right]^{2}} .
$$

This is a finite result, which is included as such in Eq. (17).

The first term of Eq. A3, on the other hand, becomes

$$
\begin{aligned}
& \frac{\mathrm{d} \sigma^{h A \rightarrow q g X}}{\mathrm{~d} y_{q} \mathrm{~d} y_{g} \mathrm{~d}^{2} \mathbf{q}_{T} \mathrm{~d}^{2} \mathbf{k}_{T}} \underset{D P}{=} x q_{h}(x) z(1-z) P_{q \rightarrow q g}(z) \\
& \alpha_{\mathrm{S}} S_{\perp} \frac{1}{(2 \pi)^{6}} \int \frac{\mathrm{d}^{2} \mathbf{n}_{T}}{\mathbf{n}_{T}{ }^{2}} 2 S\left(\mathbf{q}_{T}+\mathbf{n}_{T}\right) \widetilde{S}\left(\mathbf{k}_{T}-\mathbf{n}_{T}\right) .
\end{aligned}
$$

The interpretation of this result is illustrated in 12 the emission a gluon with transverse momentum $\mathbf{n}_{T}$ is followed by the subsequent independent scattering of the quark and the gluon off the target. The integral over $\mathbf{n}_{T}$ is logarithmically divergent in the infrared $n_{T} \rightarrow 0$. Regulating it at a parametrically confinement scale momentum $\Lambda$ we can neglect $\mathbf{n}_{T}$ compared to $\mathbf{k}_{T}, \mathbf{q}_{T}$ in the two point functions and approximate

$$
\begin{aligned}
\frac{\mathrm{d} \sigma^{h A \rightarrow q g X}}{\mathrm{~d} y_{q} \mathrm{~d} y_{g} \mathrm{~d}^{2} \mathbf{q}_{T} \mathrm{~d}^{2} \mathbf{k}_{T}} \underset{D P}{D} x q_{h}(x) z(1-z) P_{q \rightarrow q g}(z) \\
\frac{\alpha_{\mathrm{s}}}{2 \pi} \ln \Lambda^{2} \frac{1}{(2 \pi)^{4}} S\left(\mathbf{q}_{T}\right) \widetilde{S}\left(\mathbf{k}_{T}\right) .
\end{aligned}
$$

We find it instructive to compare this to the general form for the LO DGLAP evolution equation for the quarkgluon double parton distribution $D_{q g}\left(x_{q}, x_{g}, Q^{2}\right)$ (see e.g. Eq. (2.1) of Ref. [46]):

$$
\begin{aligned}
& \frac{\mathrm{d} D_{q g}\left(x_{q}, x_{g}, Q^{2}\right)}{\mathrm{d} \ln Q^{2}}=\frac{\alpha_{\mathrm{s}}\left(Q^{2}\right)}{2 \pi}[\text { evol.+ } \\
& \left.\quad q\left(x_{g}+x_{g}, Q^{2}\right) \frac{1}{x_{q}+x_{g}} P_{q \rightarrow q g}\left(\frac{x_{g}}{x_{q}+x_{g}}\right)\right] .
\end{aligned}
$$

Here "evol." denotes DGLAP evolution terms corresponding to separate radiation from quarks and gluons, leading to a variation of the double parton distribution with the scale $Q^{2}$. As we are not interested in very high transverse momentum scales we will neglect these and concentrate on the second term, which corresponds to the creation of a new correlated quark-gluon pair in the projectile due to radiation from the quark. This leads us to identify

$$
\begin{aligned}
D_{q g}\left(x_{q}, x_{g}, Q^{2}\right) \approx & \frac{\alpha_{\mathrm{s}}\left(Q^{2}\right)}{2 \pi} \ln \Lambda^{2} q\left(x_{q}+x_{g}, Q^{2}\right) \\
& \times \frac{1}{x_{q}+x_{g}} P_{q \rightarrow q g}\left(\frac{x_{g}}{x_{q}+x_{g}}\right) .
\end{aligned}
$$

With this identification we write the contribution of
Eq. A6 as

$$
\begin{array}{r}
\frac{\mathrm{d} \sigma^{h A \rightarrow q g X}}{\mathrm{~d} y_{q} \mathrm{~d} y_{g} \mathrm{~d}^{2} \mathbf{q}_{T} \mathrm{~d}^{2} \mathbf{k}_{T}} \underset{D P}{=} z(1-z) x^{2} D_{q g}\left(z x,(1-z) x, \Lambda^{2}\right) \\
\times \frac{S_{\perp}}{(2 \pi)^{4}} S\left(\mathbf{q}_{T}\right) \widetilde{S}\left(\mathbf{k}_{T}\right) . \quad \text { (A9) }
\end{array}
$$

In the uncorrelated approximation where the double parton distribution is approximated by

$$
D_{q g}\left(x_{q}, x_{g}, Q^{2}\right) \approx x_{q} q\left(x_{q}, Q^{2}\right) x_{g} g\left(x_{g}, Q^{2}\right),
$$

the result of Eq. A9 can be identified as the product of the single inclusive cross sections (1) and (2) as

$$
\frac{\mathrm{d} \sigma_{\text {DPS }}^{h A \rightarrow q g X}}{\mathrm{~d} y_{q} \mathrm{~d} y_{g} \mathrm{~d}^{2} \mathbf{q}_{T} \mathrm{~d}^{2} \mathbf{k}_{T}}=\frac{1}{S_{\perp}} \frac{\mathrm{d} \sigma^{h A \rightarrow q X}}{\mathrm{~d} y_{q} \mathrm{~d}^{2} \mathbf{q}_{T}} \frac{\mathrm{d} \sigma^{h A \rightarrow g X}}{\mathrm{~d} y_{g} \mathrm{~d}^{2} \mathbf{k}_{T}} .
$$

The prefactor, which must have the dimensions of an inverse area, is commonly denoted as $1 / \sigma_{\text {eff. }}$ In our calculation, which assumes a dilute probe hitting a large, uniform target, this misleadingly named "effective cross section" is the same as the transverse size of the target $S_{\perp}$.

\section{Appendix B: Impact parameter profiles}

Let us start (see e.g. Ref. [42]) by considering the general case of two colliding projectiles 1 and 2, of partons distributed with an impact parameter profile that we will assume is factorized into a distribution $F_{1,2}\left(\mathbf{b}_{T}\right)$ normalized as

$$
\int \mathrm{d}^{2} \mathbf{b}_{T} F_{1,2}\left(\mathbf{b}_{T}\right)=1 .
$$

We define the Fourier transform as

$$
F\left(\boldsymbol{\Delta}_{T}\right)=\int \mathrm{d}^{2} \mathbf{b}_{T} e^{i \mathbf{b}_{T} \cdot \boldsymbol{\Delta}_{T}} F\left(\boldsymbol{\Delta}_{T}\right) .
$$

A single inclusive cross section is simply

$$
\begin{aligned}
\sigma^{S} & =\int \mathrm{d}^{2} \mathbf{b}_{T 1} \mathrm{~d}^{2} \mathbf{b}_{T 2} F_{1}\left(\mathbf{b}_{T 1}\right) F_{2}\left(\mathbf{b}_{T 2}\right) \sigma^{i j} \\
& =\int \mathrm{d}^{2} \mathbf{b}_{T} \mathrm{~d}^{2} \mathbf{b}_{T 1} F_{1}\left(\mathbf{b}_{T 1}\right) F_{2}\left(\mathbf{b}_{T}-\mathbf{b}_{T 1}\right) \sigma^{i j},
\end{aligned}
$$

where $\sigma^{i j}$ is the parton level cross section multiplied by the appropriate parton distribution (which we assumed factorized from the impact parameter profile) and $\mathbf{b}_{T}$ the impact parameter of the collision. For a double scattering the cross section can be written as

$$
\begin{aligned}
\sigma^{D}=\int & \mathrm{d}^{2} \mathbf{b}_{T} \mathrm{~d}^{2} \mathbf{b}_{T 1} \mathrm{~d}^{2} \mathbf{b}_{T 1}^{\prime} F_{1}\left(\mathbf{b}_{T 1}\right) F_{1}\left(\mathbf{b}_{T 1}^{\prime}\right) \\
& F_{2}\left(\mathbf{b}_{T}-\mathbf{b}_{T 1}\right) F_{2}\left(\mathbf{b}_{T}-\mathbf{b}_{T 1}^{\prime}\right) \sigma^{i j} \sigma^{k l} \\
= & \int \frac{\mathrm{d}^{2} \boldsymbol{\Delta}_{T}}{(2 \pi)^{2}}\left|F_{1}\left(\boldsymbol{\Delta}_{T}\right)\right|^{2}\left|F_{2}\left(\boldsymbol{\Delta}_{T}\right)\right|^{2} \sigma^{i j} \sigma^{k l} .
\end{aligned}
$$


The term "effective cross section" refers to the factor

$$
\frac{1}{\sigma_{\text {eff }}}=\int \frac{\mathrm{d}^{2} \boldsymbol{\Delta}_{T}}{(2 \pi)^{2}}\left|F_{1}\left(\boldsymbol{\Delta}_{T}\right)\right|^{2}\left|F_{2}\left(\boldsymbol{\Delta}_{T}\right)\right|^{2},
$$

and has experimentally been measured as $\sigma_{\text {eff }} \approx 15 \mathrm{mb}$ in proton-antiproton collisions [47, 48. The value $15 \mathrm{mb}$ is surprisingly small (i.e. the cross section surprisingly large) compared to other estimates of the proton impact parameter profile, which could lead one to question whether it includes some additional dynamical correlations that should properly be included in the double parton distribution itself. For example a Gaussian parametrization of the TOTEM [49] $B=23.6 \mathrm{GeV}^{-2}$ elastic cross section $\mathrm{d} \sigma / \mathrm{d} t \sim e^{23.6 t / \mathrm{GeV}^{2}}$ would correspond to $F\left(\boldsymbol{\Delta}_{T}\right)=e^{-5.9 \boldsymbol{\Delta}_{T}{ }^{2} / \mathrm{GeV}^{2}}$ and $\sigma_{\text {eff }} \gtrsim 100 \mathrm{mb}$. A perhaps more relevant data point for hard small $x$ processes would be diffractive vector meson production in DIS [50, 51, where we can parametrize $F\left(\boldsymbol{\Delta}_{T}\right)=$ $e^{-B_{D} \boldsymbol{\Delta}_{T}^{2} / 2}$ with $B_{D} \approx 4 \mathrm{GeV}^{-2}$, leading to $\sigma_{\text {eff }} \approx 39 \mathrm{mb}$. The dipole parametrization estimate 52 for the proton two gluon form factor, $\left.F_{2 g}\left(\boldsymbol{\Delta}_{T}\right)=1 /\left(\boldsymbol{\Delta}_{T}{ }^{2} / m_{g}^{2}\right)+1\right)^{2}$ with $m_{g}^{2}=1.1 \mathrm{GeV}^{2}$, similarly leads to a large value for $\sigma_{\text {eff. }}$

It was, however, noticed in Ref. 40, that the experimental result $15 \mathrm{mb}$ could be interpreted as the large $x$ valence-like partons being completely localized in the transverse plane. In this case the dilute probe form factor would be $F_{1}\left(\boldsymbol{\Delta}_{T}\right)=1$ and

$$
\frac{1}{\sigma_{\text {eff }}}=\int \frac{\mathrm{d}^{2} \boldsymbol{\Delta}_{T}}{(2 \pi)^{2}}\left|F_{2}\left(\boldsymbol{\Delta}_{T}\right)\right|^{2}=\int \mathrm{d}^{2} \mathbf{b}_{T} F_{2}\left(\mathbf{b}_{T}\right)^{2} .
$$

Equation (B6) leads to an intuitive picture of the double inclusive cross section as an integral over the transverse plane of the target that is directly generalizable to nuclei. For a nuclear target with $F_{2}\left(\mathbf{b}_{T}\right) \sim T_{A}\left(\mathbf{b}_{T}\right)$ this would lead to a similar impact parameter dependence as the contributions (b) and (c) in Ref. 40. Here the target is proportional to $T_{A}\left(\mathbf{b}_{T}\right)$ only in the dilute limit and assuming that the target density is $\sim T_{A}$ would be inconsistent with a nuclear modification factor $R_{p A}$ significantly different from one in single inclusive scattering. However, motivated by the observation in Ref. [40, that the proton-proton result of $\sigma_{\text {eff }} \approx 15 \mathrm{mb}$ is consistent with the large $x$ projectile being pointlike we shall keep the interpretation (B6) for $\sigma_{\text {eff }}$. Thus the only impact parameter dependence in our calculation is an integral over the transverse profile of the target, which disappears when calculating yields instead of cross sections, as in Eqs. 11, 2), (17) and 20.
[1] F. Gelis, E. Iancu, J. Jalilian-Marian and R. Venugopalan, Ann. Rev. Nucl. Part. Sci. 60 (2010) 463 arXiv:1002.0333 [hep-ph].

[2] T. Lappi, Int. J. Mod. Phys. E20 (2011) 1 arXiv:1003.1852 [hep-ph].

[3] A. H. Mueller, Phys. Lett. B523 (2001) 243 arXiv: hep-ph/0110169.

[4] I. Balitsky, Nucl. Phys. B463 (1996) 99 arXiv: hep-ph/9509348.

[5] Y. V. Kovchegov, Phys. Rev. D60 (1999) 034008 arXiv:hep-ph/9901281.

[6] Y. V. Kovchegov, Phys. Rev. D61 (2000) 074018 arXiv:hep-ph/9905214.

[7] A. Krasnitz and R. Venugopalan, Nucl. Phys. B557 (1999) 237 arXiv:hep-ph/9809433.

[8] T. Lappi, Phys. Lett. B703 (2011) 325 arXiv:1105.5511 [hep-ph].

[9] C. Marquet and H. Weigert, Nucl. Phys. A843 (2010) 68 arXiv:1003.0813 [hep-ph].

[10] E. Braidot, arXiv:1102.0931 [nucl-ex]

[11] PHENIX collaboration, A. Adare et. al., Phys. Rev. Lett. 107 (2011) 172301 arXiv:1105.5112 [nucl-ex].

[12] PHENIX collaboration, S. S. Adler et. al., Phys. Rev. Lett. 96 (2006) 222301 arXiv:nucl-ex/0603017.

[13] PHENIX collaboration, Z. Citron, Nucl. Phys. A830 (2009) 607c arXiv:0907.4796 [nucl-ex]].

[14] STAR collaboration, E. Braidot, Nucl. Phys. A830 (2009) 603c arXiv:0907.3473 [nucl-ex].

[15] A. Dumitru and J. Jalilian-Marian, Phys. Rev. D81 (2010) 094015 arXiv:1001.4820 [hep-ph].

[16] A. Dumitru and J. Jalilian-Marian, Phys. Rev. D82
(2010) 074023 arXiv:1008.0480 [hep-ph].

[17] A. Dumitru, J. Jalilian-Marian, T. Lappi, B. Schenke and R. Venugopalan, Phys. Lett. B706 (2011) 219 arXiv: 1108.4764 [hep-ph].

[18] E. Iancu and D. Triantafyllopoulos, JHEP 1111 (2011) 105 arXiv:1109.0302 [hep-ph].

[19] E. Iancu and D. Triantafyllopoulos, JHEP 1204 (2012) 025 arXiv:1112.1104 [hep-ph].

[20] A. Kovner and M. Lublinsky, Phys. Rev. D84 (2011) 094011 arXiv:1109.0347 [hep-ph].

[21] A. Kovner and M. Lublinsky, Phys. Rev. D83 (2011) 034017 arXiv:1012.3398 [hep-ph].

[22] C. Marquet, Nucl. Phys. A796 (2007) 41 arXiv:0708.0231 [hep-ph].

[23] J. L. Albacete and C. Marquet, Phys. Rev. Lett. 105 (2010) 162301 arXiv:1005.4065 [hep-ph].

[24] F. Dominguez, C. Marquet, B.-W. Xiao and F. Yuan, Phys. Rev. D83 (2011) 105005 arXiv:1101.0715 [hep-ph].

[25] A. Stasto, B.-W. Xiao and F. Yuan, Phys. Lett. B716 (2012) 430 arXiv:1109.1817 [hep-ph].

[26] STAR collaboration, J. Adams et. al., Phys. Rev. Lett. 97 (2006) 152302 arXiv:nucl-ex/0602011.

[27] BRAHMS collaboration, I. Arsene et. al., Phys. Rev. Lett. 93 (2004) 242303 arXiv:nucl-ex/0403005.

[28] I. Balitsky, Phys. Rev. D75 (2007) 014001 arXiv:hep-ph/0609105.

[29] J. L. Albacete and Y. V. Kovchegov, Phys. Rev. D75 (2007) 125021 arXiv:0704.0612 [hep-ph].

[30] A. Dumitru and J. Jalilian-Marian, Phys. Lett. B547 (2002) 15 arXiv:hep-ph/0111357. 
[31] A. Dumitru and J. Jalilian-Marian, Phys. Rev. Lett. 89 (2002) 022301 arXiv:hep-ph/0204028.

[32] A. Dumitru, A. Hayashigaki and J. Jalilian-Marian, Nucl. Phys. A765 (2006) 464 arXiv:hep-ph/0506308.

[33] H.-L. Lai, M. Guzzi, J. Huston, Z. Li, P. M. Nadolsky et. al., Phys. Rev. D82 (2010) 074024 arXiv:1007.2241 [hep-ph].

[34] D. de Florian, R. Sassot and M. Stratmann, Phys. Rev. D75 (2007) 114010 arXiv:hep-ph/0703242 [HEP-PH].

[35] J. L. Albacete and C. Marquet, Phys.Lett. B687 (2010) 174 arXiv:1001.1378 [hep-ph].

[36] H. Kowalski, T. Lappi and R. Venugopalan, Phys. Rev. Lett. 100 (2008) 022303 arXiv:0705.3047 [hep-ph].

[37] P. Tribedy and R. Venugopalan, Phys.Lett. B710 (2012) 125 arXiv:1112.2445 [hep-ph].

[38] H. Fujii, K. Itakura, Y. Kitadono and Y. Nara, J. Phys. $G$ G38 (2011) 124125 arXiv: 1107.1333 [hep-ph].

[39] J. L. Albacete and A. Dumitru, arXiv:1011.5161 [hep-ph]

[40] M. Strikman and W. Vogelsang, Phys. Rev. D83 (2011) 034029 arXiv: 1009.6123 [hep-ph].

[41] M. Strikman and D. Treleani, Phys. Rev. Lett. 88 (2002) 031801 arXiv:hep-ph/0111468 [hep-ph].

[42] B. Blok, Y. Dokshitzer, L. Frankfurt and M. Strikman,
Phys. Rev. D83 (2011) 071501 arXiv: 1009.2714 [hep-ph].

[43] K. Tuchin, Nucl. Phys. A846 (2010) 83 arXiv:0912.5479 [hep-ph].

[44] Y. V. Kovchegov, J. Kuokkanen, K. Rummukainen and H. Weigert, Nucl. Phys. A823 (2009) 47 arXiv:0812.3238 [hep-ph].

[45] STAR collaboration, C. Perkins, arXiv:1109.0649 [nucl-ex]

[46] J. R. Gaunt and W. J. Stirling, JHEP 1003 (2010) 005 arXiv:0910.4347 [hep-ph].

[47] CDF collaboration, F. Abe et. al., Phys. Rev. D56 (1997) 3811.

[48] Do collaboration, V. Abazov et. al., Phys. Rev. D81 (2010) 052012 arXiv:0912.5104 [hep-ex].

[49] TOTEM collaboration, G. Antchev et. al., Europhys. Lett. 95 (2011) 41001 [arXiv:1110.1385 [hep-ex]].

[50] H. Kowalski, L. Motyka and G. Watt, Phys. Rev. D74 (2006) 074016 arXiv:hep-ph/0606272.

[51] T. Lappi and H. Mantysaari, Phys. Rev. C83 (2011) 065202 arXiv:1011.1988 [hep-ph].

[52] L. Frankfurt and M. Strikman, Phys. Rev. D66 (2002) 031502 arXiv:hep-ph/0205223 [hep-ph]. 